\title{
A minimal model of neutrino flavor
}

\author{
Christoph Luhn, ${ }^{a}$ Krishna Mohan Parattu ${ }^{b}$ and Akın Wingerter ${ }^{c}$ \\ ${ }^{a}$ Institute for Particle Physics Phenomenology, University of Durham, \\ Durham DH1 3LE, U.K. \\ ${ }^{b}$ Inter-University Centre for Astronomy and Astrophysics, \\ Ganeshkhind, Pune 41100\%, India \\ ${ }^{c}$ Laboratoire de Physique Subatomique et de Cosmologie, UJF Grenoble 1, CNRS/IN2P3, INPG, \\ 53 Avenue des Martyrs, F-38026 Grenoble, France \\ E-mail: christoph.luhn@durham.ac.uk, krishna@iucaa.ernet.in, \\ akin@lpsc.in2p3.fr
}

ABSTRACT: Models of neutrino mass which attempt to describe the observed lepton mixing pattern are typically based on discrete family symmetries with a non-Abelian and one or more Abelian factors. The latter so-called shaping symmetries are imposed in order to yield a realistic phenomenology by forbidding unwanted operators. Here we propose a supersymmetric model of neutrino flavor which is based on the group $T_{7}$ and does not require extra $\mathbb{Z}_{N}$ or U(1) factors in the Yukawa sector, which makes it the smallest realistic family symmetry that has been considered so far. At leading order, the model predicts tribimaximal mixing which arises completely accidentally from a combination of the $T_{7}$ Clebsch-Gordan coefficients and suitable flavon alignments. Next-to-leading order (NLO) operators break the simple tribimaximal structure and render the model compatible with the recent results of the Daya Bay and Reno collaborations which have measured a reactor angle of around $9^{\circ}$. Problematic NLO deviations of the other two mixing angles can be controlled in an ultraviolet completion of the model. The vacuum alignment mechanism that we use necessitates the introduction of a hidden flavon sector that transforms under a $\mathbb{Z}_{6}$ symmetry, thereby spoiling the minimality of our model whose flavor symmetry is then $T_{7} \times \mathbb{Z}_{6}$.

Keywords: Neutrino Physics, Discrete and Finite Symmetries, Beyond Standard Model

ArXiv EPRINT: 1210.1197 


\section{Contents}

1 Introduction 1

2 The minimal $T_{7}$ model $\quad 3$

2.1 Tribimaximal mixing at leading order 3

2.2 Next-to-leading order effects 5

$\begin{array}{lll}2.3 & \text { Phenomenology } & 7\end{array}$

3 An ultraviolet completion of the model $\quad 8$

$\begin{array}{lll}3.1 & \text { The charged lepton sector } & 10\end{array}$

$\begin{array}{lll}3.2 & \text { The neutrino sector } & 11\end{array}$

4 Vacuum alignment $\quad 14$

$\begin{array}{lll}4.1 & \text { General discussion } & 14\end{array}$

$\begin{array}{lll}4.2 & \text { Alignment through a hidden flavon sector } & 15\end{array}$

$\begin{array}{llr}5 & \text { Conclusions } & 18\end{array}$

$\begin{array}{ll}\text { A Generators and Clebsch-Gordan coefficients of } T_{7} & 19\end{array}$

\section{Introduction}

The triplication of chiral families remains one of the biggest mysteries in particle physics. A clue towards unraveling the principle behind the fact that quarks and leptons come in three copies is provided by the observation of a remarkable lepton mixing pattern: contrary to the quark sector, the mixing of the leptons is described by two large and one small angle. Until recently, the results of neutrino oscillation experiments were well compatible with a PMNS matrix of the intriguingly simple structure [1-3]

$$
U_{\mathrm{PMNS}} \approx\left(\begin{array}{ccc}
\frac{2}{\sqrt{6}} & \frac{1}{\sqrt{3}} & 0 \\
-\frac{1}{\sqrt{6}} & \frac{1}{\sqrt{3}} & \frac{1}{\sqrt{2}} \\
-\frac{1}{\sqrt{6}} & \frac{1}{\sqrt{3}} & -\frac{1}{\sqrt{2}}
\end{array}\right) .
$$

This so-called tribimaximal mixing suggests an intimate connection of the three generations of leptons, which can be realized in the framework of non-Abelian discrete family symmetries. Imposing such a horizontal symmetry $G$ allows to unify different generations into a multiplet of the given non-Abelian group. With three families, the physically interesting groups should have a triplet representation, limiting possible choices to subgroups of U(3). Many of these have been successfully applied to construct models of tribimaximal lepton mixing, see for instance [4-6] and references therein. 
In general, family symmetry models can be classified according to the origin of the symmetry of the neutrino mass matrix. We will assume that neutrinos are Majorana particles; then their mass matrix is always symmetric under a Klein symmetry $\mathbb{Z}_{2} \times \mathbb{Z}_{2}$. Working in a basis where the charged leptons are diagonal, ${ }^{1}$ the explicit form of the Klein symmetry expressed in terms of $3 \times 3$ matrices is dictated by the PMNS mixing matrix and can be determined as

$$
U_{\mathrm{PMNS}}^{*}\left(\begin{array}{ccc}
(-1)^{p} & 0 & 0 \\
0 & (-1)^{q} & 0 \\
0 & 0 & (-1)^{p+q}
\end{array}\right) U_{\mathrm{PMNS}}^{T} \cdot
$$

Here $p=0,1$ and $q=0,1$, yielding a symmetry group of four elements. This neutrino flavor symmetry can arise as a residual symmetry of the underlying family symmetry $G$, in other words, the four elements of eq. (1.2) can also be elements of the imposed family symmetry. Models of this type are called direct models [14]. In indirect models, on the other hand, the above Klein symmetry is not a subgroup of $G$. Models of this class are typically based on the type I seesaw mechanism [15-18] with the assumption of sequential dominance [19-21], i.e. a hierarchy among the three terms arising from three right-handed neutrinos. Here, the main role of the family symmetry consists in explaining special vacuum configurations of the flavon fields that break the family symmetry [14]. In addition to these pure classes of models there are semi-direct models in which one of the $\mathbb{Z}_{2}$ factors of the Klein symmetry arises as a residual symmetry of $G$, while the other factor arises accidentally. In fact, the famous Altarelli-Feruglio model based on $A_{4}[22,23]$ belongs to the semi-direct class as the tribimaximal $\mu-\tau$ symmetry is not part of $A_{4}$.

Hitherto, regardless of the type of model, the non-Abelian family symmetry has always been augmented by extra Abelian factors such as $\mathbb{Z}_{N}$ and $\mathrm{U}(1)$ in order to yield realistic phenomenology. These shaping symmetries were crucial for controlling the coupling of the Standard Model neutral flavon fields to the leptons. In [22, 23], for instance, the neutrino and charged lepton sectors are separated by means of a $\mathbb{Z}_{3}$ shaping symmetry. In that sense, one should speak more precisely of the Altarelli-Feruglio $A_{4} \times \mathbb{Z}_{3}$ model, and the family symmetry $G$ is thus a group of order 36, rather than 12 .

Mindful of this subtlety of defining the full family symmetry of a model, a systematic scan over 76 different groups has been performed in [24] with the purpose of studying whether or not there is an inherent connection between $A_{4}$ and tribimaximal mixing. The results of the scan proved that there are indeed several, even more minimal, groups which are capable of describing tribimaximal mixing (assuming the imposed simple alignment of the flavon vevs can be justified). The smallest such group ${ }^{2}$ was identified to be $T_{7}$, a group

\footnotetext{
${ }^{1}$ This classification can also be applied to GUT models where the charged lepton mass matrix is typically only approximately diagonal. In such a setup, the total PMNS mixing matrix will involve charged lepton corrections that have to be taken into account separately, see e.g. [7-11], leading to characteristic mixing sum rules $[12,13]$.

${ }^{2}$ As we will see in section 4 , introducing a dynamical mechanism for vacuum alignment will necessitate to augment the family symmetry by an extra $\mathbb{Z}_{6}$ factor. As such, our symmetry group $T_{7} \times \mathbb{Z}_{6}$ will be larger than $A_{4} \times \mathbb{Z}_{3}$ that was used in [22, 23]. Note, however, that the $\mathbb{Z}_{3}$ factor in $[22,23]$ is needed not only for the flavon alignment, but also to control the coupling of the flavon fields to the neutrino or charged lepton sector, respectively, which in our case can be achieved by $T_{7}$ alone.
} 
of order 21 which is sometimes also called the Frobenius group $\mathbb{Z}_{7} \rtimes \mathbb{Z}_{3}$ [25-27]. Note that this non-Abelian symmetry does not require any extra shaping symmetry. It is furthermore interesting to point out that $T_{7}$ has no $\mathbb{Z}_{2}$ subgroups, and hence the Klein symmetry of the neutrino mass matrix cannot be part of this family symmetry. As such, a corresponding $T_{7}$ model would be neither direct nor semi-direct, but rather of indirect type. Not necessarily requiring the seesaw mechanism, it would - to the best of our knowledge - be the first indirect model which is not based on the assumption of sequential dominance and the quadratic appearance of flavon fields in the effective neutrino mass term.

It is the purpose of this article to present the details of a complete $T_{7}$ model of leptons which yields tribimaximal mixing at leading order, including a discussion of the vacuum alignment. We put particular emphasis on explaining how the Klein symmetry arises completely accidentally from a combination of the $T_{7}$ Clebsch-Gordan (CG) coefficients and suitable flavon alignments. As tribimaximal mixing has been ruled out by the Daya Bay [28] and Reno [29] measurements of a reactor mixing angle $\theta_{13}$ of around $9^{\circ}$, it is necessary to investigate if next-to-leading order (NLO) effects can generate large enough deviations from the tribimaximal leading order prediction. With the Klein symmetry arising purely accidentally, NLO terms are bound to perturb the tribimaximal mixing pattern. While switching on $\theta_{13}$, NLO corrections will in general also give rise to perturbations of the atmospheric and, more critically, the solar mixing angle which may be phenomenologically unacceptable. However, these unwanted NLO corrections can be suppressed in an ultraviolet completed version of the $T_{7}$ model involving special messenger fields.

The remainder of this article is organized as follows. We define and discuss the minimal $T_{7}$ model in section 2 , both at leading as well as next-to-leading order. The ultraviolet completion of the model is presented in section 3. Section 4 addresses the question of vacuum alignment, and the conclusions are drawn in section 5 . The relevant group theoretic details of $T_{7}$ are laid out in appendix A, including the generators of the representations in a basis with a diagonal order three element as well as the corresponding CG coefficients.

\section{The minimal $T_{7}$ model}

\subsection{Tribimaximal mixing at leading order}

In this section we describe a minimal model of leptons based on the family symmetry $T_{7}$. This non-Abelian finite group comprises 21 elements and has three singlet as well as two triplet representations, which we denote by $\mathbf{1}, \mathbf{1}^{\prime}, \mathbf{1}^{\prime \prime}, \mathbf{3}$ and $\overline{\mathbf{3}}$, respectively. As the model does not feature any shaping symmetries, its structure is solely determined by the family symmetry $T_{7}$ as well as the gauge symmetry $\mathrm{SU}(2)_{L} \times \mathrm{U}(1)_{Y}$. We work in a supersymmetric framework with two Higgs doublets, $h_{u}$ and $h_{d}$, transforming trivially under $T_{7}$. The three generations of left-handed lepton doublets $L$ are unified in a triplet representation of $T_{7}$, while the right-handed charged leptons $e^{c}, \mu^{c}$ and $\tau^{c}$ live in the three distinct onedimensional representations. In order to break the family symmetry, two flavon fields are introduced, namely $\widetilde{\varphi}$ and $\varphi$ transforming as a $\overline{\mathbf{3}}$ and $\mathbf{3}$, respectively. The particle content of this model is summarized in table 1 , where we have also listed the standard charge assignments under hypercharge $\mathrm{U}(1)_{Y}$ and $\mathrm{U}(1)_{R}$ for convenience. 


\begin{tabular}{|c||c|c|c|c|c|c||c|c|}
\hline Field & $L$ & $e^{c}$ & $\mu^{c}$ & $\tau^{c}$ & $h_{u}$ & $h_{d}$ & $\widetilde{\varphi}$ & $\varphi$ \\
\hline$T_{7}$ & $\mathbf{3}$ & $\mathbf{1}$ & $\mathbf{1}^{\prime}$ & $\mathbf{1}^{\prime \prime}$ & $\mathbf{1}$ & $\mathbf{1}$ & $\mathbf{3}$ & $\mathbf{3}$ \\
\hline $\mathrm{U}(1)_{Y}$ & -1 & 2 & 2 & 2 & 1 & -1 & 0 & 0 \\
\hline $\mathrm{U}(1)_{R}$ & 1 & 1 & 1 & 1 & 0 & 0 & 0 & 0 \\
\hline
\end{tabular}

Table 1. The transformation properties of the minimal $T_{7}$ model of leptons.

With these $T_{7}$ assignments, the leading order superpotentials of the charged lepton and the neutrino sector read

$$
\begin{aligned}
& W_{\ell}=y_{e} \frac{\widetilde{\varphi}}{\Lambda} L e^{c} h_{d}+y_{\mu} \frac{\widetilde{\varphi}}{\Lambda} L \mu^{c} h_{d}+y_{\tau} \frac{\widetilde{\varphi}}{\Lambda} L \tau^{c} h_{d}, \\
& W_{\nu}=y_{1} \frac{\widetilde{\varphi}}{\Lambda^{2}} L L h_{u} h_{u}+y_{2} \frac{\varphi}{\Lambda^{2}} L L h_{u} h_{u},
\end{aligned}
$$

respectively. Here, the $y$ 's are dimensionless coupling constants, and $\Lambda$ denotes a common cut-off above the scale of family symmetry breaking. The neutrino mass terms originate from the Weinberg operator [30] augmented by a flavon field. The coefficients $y_{e}, y_{\mu}$, $y_{\tau}$ correspond to the Yukawa couplings of the charged leptons which we will assume to be hierarchical. Note that one can naturally generate this hierarchy by introducing an additional Froggatt-Nielsen U(1) $)_{\mathrm{FN}}$ under which the flavon fields are charged [31]. For an example of a model that realizes hierarchical charged lepton masses with an $A_{4} \times \mathbb{Z}_{4}$ flavor symmetry alone and without invoking a $\mathrm{U}(1)_{\mathrm{FN}}$, see [32].

It is important to note that only one flavon, $\widetilde{\varphi}$, enters the charged lepton sector at leading order. The other flavon, $\varphi$, cannot couple to the charged leptons due to the absence of a singlet representation in the $T_{7}$ Kronecker product $\mathbf{3} \otimes \mathbf{3}$, see appendix A. We will see below that the vev of $\widetilde{\varphi}$ can be chosen such that it breaks $T_{7}$ down to $\mathbb{Z}_{3}$, thus leading to a diagonal charged lepton mass matrix. In the neutrino sector, both flavons are present, as the symmetric product of $\mathbf{3} \otimes \mathbf{3}$ contains both a $\mathbf{3}$ and a $\overline{\mathbf{3}}$.

In order to find the vacuum alignments that lead to tribimaximal mixing, we insert the flavon vevs in eq. (2.1) and eq. (2.2) in their most general form. Contracting the $T_{7}$ indices using the CG coefficients given in appendix A and inserting the Higgs vevs $v_{u}$ and $v_{d}$, we obtain the respective mass matrices. In the left-right convention for the charged leptons, where the left-handed particles are to the left of the Yukawa matrix, we find

$$
\begin{aligned}
M_{\ell}= & {\left[\left(\begin{array}{ccc}
y_{e} & 0 & 0 \\
0 & y_{\mu} & 0 \\
0 & 0 & y_{\tau}
\end{array}\right) \frac{\left\langle\widetilde{\varphi}_{1}\right\rangle}{\Lambda}+\left(\begin{array}{ccc}
0 & 0 & y_{\tau} \\
y_{e} & 0 & 0 \\
0 & y_{\mu} & 0
\end{array}\right) \frac{\left\langle\widetilde{\varphi}_{2}\right\rangle}{\Lambda}+\left(\begin{array}{ccc}
0 & y_{\mu} & 0 \\
0 & 0 & y_{\tau} \\
y_{e} & 0 & 0
\end{array}\right) \frac{\left\langle\widetilde{\varphi}_{3}\right\rangle}{\Lambda}\right] \frac{v_{d}}{\sqrt{3}}, } \\
M_{\nu}= & y_{1}\left[\left(\begin{array}{lll}
1 & 0 & 0 \\
0 & 0 & 1 \\
0 & 1 & 0
\end{array}\right) \frac{\left\langle\widetilde{\varphi}_{1}\right\rangle}{\Lambda}+\left(\begin{array}{ccc}
0 & \omega & 0 \\
\omega & 0 & 0 \\
0 & 0 & \omega
\end{array}\right) \frac{\left\langle\widetilde{\varphi}_{2}\right\rangle}{\Lambda}+\left(\begin{array}{ccc}
0 & 0 & \omega^{2} \\
0 & \omega^{2} & 0 \\
\omega^{2} & 0 & 0
\end{array}\right) \frac{\left\langle\widetilde{\varphi}_{3}\right\rangle}{\Lambda}\right] \frac{v_{u}^{2}}{3 \Lambda} \\
& +y_{2}\left[\left(\begin{array}{ccc}
2 & 0 & 0 \\
0 & 0 & -1 \\
0 & -1 & 0
\end{array}\right) \frac{\left\langle\varphi_{1}\right\rangle}{\Lambda}+\left(\begin{array}{ccc}
0 & 0 & -1 \\
0 & 2 & 0 \\
-1 & 0 & 0
\end{array}\right) \frac{\left\langle\varphi_{2}\right\rangle}{\Lambda}+\left(\begin{array}{ccc}
0 & -1 & 0 \\
-1 & 0 & 0 \\
0 & 0 & 2
\end{array}\right)\right.
\end{aligned}
$$


This simple pattern suggests the following flavon vacuum configuration to obtain tribimaximal mixing:

$$
\langle\widetilde{\varphi}\rangle=v_{\widetilde{\varphi}}\left(\begin{array}{l}
1 \\
0 \\
0
\end{array}\right), \quad\langle\varphi\rangle=v_{\varphi} \frac{1}{\sqrt{3}}\left(\begin{array}{l}
1 \\
1 \\
1
\end{array}\right) .
$$

The alignment of $\widetilde{\varphi}$ ensures a diagonal charged lepton mass matrix in eq. (2.3), and brings the first line in eq. (2.4) into tribimaximal form. The alignment of $\varphi$ is of an extremely simple form and generates a contribution to $M_{\nu}$ which, again, has tribimaximal structure. Adopting the flavon alignments of eq. (2.5), our minimal $T_{7}$ model therefore predicts the following charged lepton and neutrino mass matrices at leading order,

$$
\begin{aligned}
& M_{\ell}=\left(\begin{array}{ccc}
y_{e} & 0 & 0 \\
0 & y_{\mu} & 0 \\
0 & 0 & y_{\tau}
\end{array}\right) \frac{v_{\tilde{\varphi}}}{\Lambda} \frac{v_{d}}{\sqrt{3}}, \\
& M_{\nu}=\left[y_{1}\left(\begin{array}{lll}
1 & 0 & 0 \\
0 & 0 & 1 \\
0 & 1 & 0
\end{array}\right) \frac{v_{\tilde{\varphi}}}{\Lambda}+y_{2}\left(\begin{array}{ccc}
2 & -1 & -1 \\
-1 & 2 & -1 \\
-1 & -1 & 2
\end{array}\right) \frac{v_{\varphi}}{\sqrt{6} \Lambda}\right] \frac{v_{u}^{2}}{3 \Lambda},
\end{aligned}
$$

implying a tribimaximal PMNS mixing matrix and the complex valued neutrino masses

$$
\left(m_{1}^{\nu}, m_{2}^{\nu}, m_{3}^{\nu}\right)=\left(2 y_{1} v_{\widetilde{\varphi}}+\sqrt{6} y_{2} v_{\varphi}, 2 y_{1} v_{\widetilde{\varphi}},-2 y_{1} v_{\widetilde{\varphi}}+\sqrt{6} y_{2} v_{\varphi}\right) \frac{v_{u}^{2}}{6 \Lambda^{2}}
$$

Before continuing to the study of NLO effects, let us pause for a moment and appreciate the beauty of the leading order model. With regard to the charged lepton sector, we observe that the alignment of $\widetilde{\varphi}$ is left invariant by the diagonal order-three generator $d$ of $T_{7}$, see table 5 in appendix $\mathrm{A}$. The corresponding $\mathbb{Z}_{3}$ symmetry which is thus preserved in the charged lepton sector after the family symmetry is broken enforces a diagonal $M_{\ell}$. In contrast, the tribimaximal Klein symmetry $\mathbb{Z}_{2} \times \mathbb{Z}_{2}$ of $M_{\nu}$ arises completely accidentally from a combination of the $T_{7} \mathrm{CG}$ coefficients and suitable flavon alignments (which will be derived and thus justified in section 4). This situation is reminiscent of the AltarelliFeruglio model $[22,23]$ where one $\mathbb{Z}_{2}$ is contained in $A_{4}$ while the other $\mathbb{Z}_{2}$, corresponding to a $\mu-\tau$ symmetry, arises accidentally. In the case of $T_{7}$, none of the two $\mathbb{Z}_{2}$ factors of the tribimaximal Klein symmetry is a part of the underlying family symmetry. As a consequence, one expects no protection of the tribimaximal structure when NLO terms are taken into account.

\section{$2.2 \quad$ Next-to-leading order effects}

The next-to-leading order superpotential for the charged and neutral leptons contains all terms up to mass dimension six and seven, respectively, that are invariant under the gauge and family symmetries. Here, we only indicate the terms that are not already part of the 
leading-order superpotential:

$$
\begin{gathered}
\Delta W_{\ell}=\frac{1}{\Lambda^{2}}\left(C_{1}^{e} L e^{c} h_{d} \varphi \varphi+C_{2}^{e} L e^{c} h_{d} \varphi \widetilde{\varphi}+C_{3}^{e} L e^{c} h_{d} \widetilde{\varphi} \widetilde{\varphi}+\right. \\
C_{1}^{\mu} L \mu^{c} h_{d} \varphi \varphi+C_{2}^{\mu} L \mu^{c} h_{d} \varphi \widetilde{\varphi}+C_{3}^{\mu} L \mu^{c} h_{d} \widetilde{\varphi} \widetilde{\varphi}+ \\
\left.C_{1}^{\tau} L \tau^{c} h_{d} \varphi \varphi+C_{2}^{\tau} L \tau^{c} h_{d} \varphi \widetilde{\varphi}+C_{3}^{\tau} L \tau^{c} h_{d} \widetilde{\varphi} \widetilde{\varphi}\right) \\
\Delta W_{\nu}=\frac{1}{\Lambda^{3}}\left(C_{1}^{\nu}(L L)_{3} h_{u} h_{u} \varphi \varphi+C_{2}^{\nu}(L L)_{\overline{3}} h_{u} h_{u} \varphi \varphi+C_{3}^{\nu}(L L)_{3} h_{u} h_{u} \widetilde{\varphi} \widetilde{\varphi}+\right. \\
\left.C_{4}^{\nu}(L L)_{\overline{3}} h_{u} h_{u} \widetilde{\varphi} \widetilde{\varphi}+C_{5}^{\nu}(L L)_{\mathbf{3}} h_{u} h_{u} \varphi \widetilde{\varphi}+C_{6}^{\nu}(L L)_{\overline{3}} h_{u} h_{u} \varphi \widetilde{\varphi}\right) .
\end{gathered}
$$

The subscripts in eq. (2.10) indicate that we have contracted the family indices of the corresponding product to obtain a $\mathbf{3}$ and $\overline{\mathbf{3}}$, respectively. As before, contracting the family and gauge indices, and substituting the vevs for the Higgs and flavon fields gives:

$$
\begin{aligned}
& \Delta M_{\ell}=\frac{v_{d}}{\Lambda^{2}} \frac{1}{3}\left[C_{1}^{e}\left(\begin{array}{lll}
0 & 0 & 0 \\
0 & 0 & 0 \\
0 & 0 & 0
\end{array}\right) v_{\varphi}^{2}+C_{2}^{e}\left(\begin{array}{ccc}
1 & 0 & 0 \\
1 & 0 & 0 \\
1 & 0 & 0
\end{array}\right) \frac{v_{\varphi}}{\sqrt{3}} v_{\widetilde{\varphi}}+C_{3}^{e}\left(\begin{array}{ccc}
1 & 0 & 0 \\
0 & 0 & 0 \\
0 & 0 & 0
\end{array}\right) v_{\widetilde{\varphi}}^{2}\right. \\
& +C_{1}^{\mu}\left(\begin{array}{lll}
0 & 0 & 0 \\
0 & 0 & 0 \\
0 & 0 & 0
\end{array}\right) v_{\varphi}^{2}+C_{2}^{\mu}\left(\begin{array}{lll}
0 & 1 & 0 \\
0 & 1 & 0 \\
0 & 1 & 0
\end{array}\right) \frac{v_{\varphi}}{\sqrt{3}} v_{\widetilde{\varphi}}+C_{3}^{\mu}\left(\begin{array}{lll}
0 & 0 & 0 \\
0 & 1 & 0 \\
0 & 0 & 0
\end{array}\right) v_{\widetilde{\varphi}}^{2} \\
& \left.+C_{1}^{\tau}\left(\begin{array}{lll}
0 & 0 & 0 \\
0 & 0 & 0 \\
0 & 0 & 0
\end{array}\right) v_{\varphi}^{2}+C_{2}^{\tau}\left(\begin{array}{lll}
0 & 0 & 1 \\
0 & 0 & 1 \\
0 & 0 & 1
\end{array}\right) \frac{v_{\varphi}}{\sqrt{3}} v_{\widetilde{\varphi}}+C_{3}^{\tau}\left(\begin{array}{lll}
0 & 0 & 0 \\
0 & 0 & 0 \\
0 & 0 & 1
\end{array}\right) v_{\widetilde{\varphi}}^{2}\right] \text {, } \\
& \Delta M_{\nu}=\frac{v_{u}^{2}}{\Lambda^{3}} \frac{1}{9 \sqrt{2}}\left[C_{1}^{\nu}\left(\begin{array}{lll}
0 & 0 & 0 \\
0 & 0 & 0 \\
0 & 0 & 0
\end{array}\right) v_{\varphi}^{2}+\sqrt{3} C_{2}^{\nu}\left(\begin{array}{ccc}
2 & -\omega^{2} & -\omega \\
-\omega^{2} & 2 \omega & -1 \\
-\omega & -1 & 2 \omega^{2}
\end{array}\right) v_{\varphi}^{2}+\sqrt{6} C_{3}^{\nu}\left(\begin{array}{lll}
1 & 0 & 0 \\
0 & 0 & 1 \\
0 & 1 & 0
\end{array}\right) v_{\widetilde{\varphi}}^{2}\right. \\
& \left.+\sqrt{6} C_{4}^{\nu}\left(\begin{array}{ccc}
2 & 0 & 0 \\
0 & 0 & -1 \\
0 & -1 & 0
\end{array}\right) v_{\widetilde{\varphi}}^{2}+\sqrt{2} C_{5}^{\nu}\left(\begin{array}{ccc}
1 & \omega & \omega^{2} \\
\omega & \omega^{2} & 1 \\
\omega^{2} & 1 & \omega
\end{array}\right) v_{\varphi} v_{\widetilde{\varphi}}+C_{6}^{\nu}\left(\begin{array}{ccc}
2 & -\omega & -\omega^{2} \\
-\omega & 2 \omega^{2} & -1 \\
-\omega^{2} & -1 & 2 \omega
\end{array}\right) v_{\varphi} v_{\widetilde{\varphi}}\right] .
\end{aligned}
$$

A quick inspection of eq. (2.11) shows that the contributions proportional to $C_{1}^{e}, C_{1}^{\mu}, C_{1}^{\tau}$ are identically zero and have no effect whatsoever on the mass matrices. The contributions proportional to $C_{3}^{e}, C_{3}^{\mu}, C_{3}^{\tau}$ do not disturb the diagonal structure of $M_{\ell}$ and hence do not affect the bi-unitary transformations that diagonalize it and that enter in the definition of $U_{\text {PMNS }}$. In contrast, $C_{2}^{e}, C_{2}^{\mu}, C_{2}^{\tau}$ will lead to a departure from tribimaximal mixing, where it has to be noted that the magnitudes of the coefficients have to be weighted by the corresponding Yukawa couplings, and hence the effect of $C_{2}^{e}$ will be negligible.

From eq. (2.12) we see that the term proportional to $C_{1}^{\nu}$ is identically zero and will not have an impact on the mixing matrix. The term corresponding to $C_{3}^{\nu}$ is non-vanishing, but commutes with the two generators of the tribimaximal Klein symmetry (see e.g. [4] for the explicit form of the generators), and as a consequence does not disturb the tribimaximal form of the mixing matrix.

The NLO terms that do cause a departure from tribimaximal mixing are those corresponding to $C_{2}^{\nu}, C_{4}^{\nu}, C_{5}^{\nu}, C_{6}^{\nu}$. The contribution proportional to $C_{4}^{\nu}$ is invariant under a $\mu-\tau$ symmetry (which corresponds to one of the generators of the tribimaximal Klein symmetry) and thus leaves $\theta_{23}$ and $\theta_{13}$ unchanged. Of particular interest is the term proportional to $C_{5}^{\nu}$, since its effect is such that $\theta_{12}$ is almost unchanged, $\theta_{23}$ stays within its $3 \sigma$ 
interval, and $\theta_{13}$ receives a sizable contribution. Therefore it seems to be a good strategy to try and suppress all NLO contributions to the superpotential except the one proportional to $C_{5}^{\nu}$. Thus, we will keep the deviation of $\theta_{12}$ and $\theta_{23}$ from the tribimaximal case small, and at the same time achieve a non-vanishing $\theta_{13}$ in agreement with experiment. In section 3 we present a renormalizable, ultraviolet completion of our model that meets exactly these criteria outlined above. Before that, however, we will explore in section 2.3 the phenomenology of our model at the effective level. We first consider the model in its most general form without any specific assumptions about the coefficients. At the end of section 2.3 we also discuss the case where all coefficients which break the tribimaximal structure, except for $C_{5}^{\nu}$, are set to zero.

\subsection{Phenomenology}

We now compare our results with experiment [28, 29, 33-35]. To that end, we first have to specify the coefficients in the leading and next-to-leading order superpotential (cf. eq. (2.1), eq. (2.2), eq. (2.9), eq. (2.10)). The mixing angles, CP phases and masses will then be uniquely determined.

The leading-order superpotential gives tribimaximal mixing which is formdiagonalizable [36]. As such, the mixing angles are in principle independent of the coefficients $y_{e}, y_{\mu}, y_{\tau}, y_{1}, y_{2}$. However, changing the coefficients affects the neutrino masses and can lead to a reordering of the mass eigenstates that in turn has an impact on the mixing angles. Since we work in a basis where the charged lepton mass matrix is diagonal, we can identify $y_{e}, y_{\mu}, y_{\tau}$ with the corresponding Yukawa couplings for which we substitute their experimentally determined values [37]. For $y_{1}$ and $y_{2}$, we choose values such that we can fit the mass ratio $\Delta m_{31}^{2} / \Delta m_{21}^{2}$.

In figure 1 we present the contour lines of $\Delta m_{31}^{2} / \Delta m_{21}^{2} \equiv 30$ as a function of $y_{1} v_{\widetilde{\varphi}}$ and $y_{2} v_{\varphi}$ (cf. eq. (2.7)) for normal neutrino mass orderings. Here we do not consider the case of inverted neutrino mass orderings as it would require $\left|y_{1} v_{\widetilde{\varphi}}\right| \ll\left|y_{2} v_{\varphi}\right|$. The green region in figure 1 corresponds to values of the parameters for which we obtain tribimaximal mixing, whereas in the red region, the mixing matrix corresponds to a permutation of the columns of $U_{\mathrm{PMNS}}$, i.e. tribimaximal mixing and the desired mass hierarchy cannot be simultaneously satisfied. Note that we have chosen $y_{1}$ and $y_{2}$ to be real for presentational purposes only. In the general case, $y_{1}$ and $y_{2}$ are complex, and to fit the mass ratio is equally easy.

In the next-to-leading order superpotential, we assume that the fundamental scale of the theory is about one order of magnitude larger than the flavon vevs and fix the ratio at $v_{\varphi} / \Lambda=v_{\widetilde{\varphi}} / \Lambda=0.10$ (see eq. (2.11) and eq. (2.12)). For the coefficients $C_{1}^{\nu}, \ldots, C_{3}^{\tau}$, we choose 1000 sets of complex numbers whose real and imaginary parts are uniformly distributed between -2.5 and +2.5 . For each such set, we calculate the corrections to the charged lepton and neutrino mass matrices and recalculate the mixing angles and masses. Note that now, $M_{\ell}$ is not necessarily diagonal, and we have to resort to the general definition of $U_{\text {PMNS }}$ in terms of the bi-unitary transformations that diagonalize the charged lepton and neutrino mass matrices. 


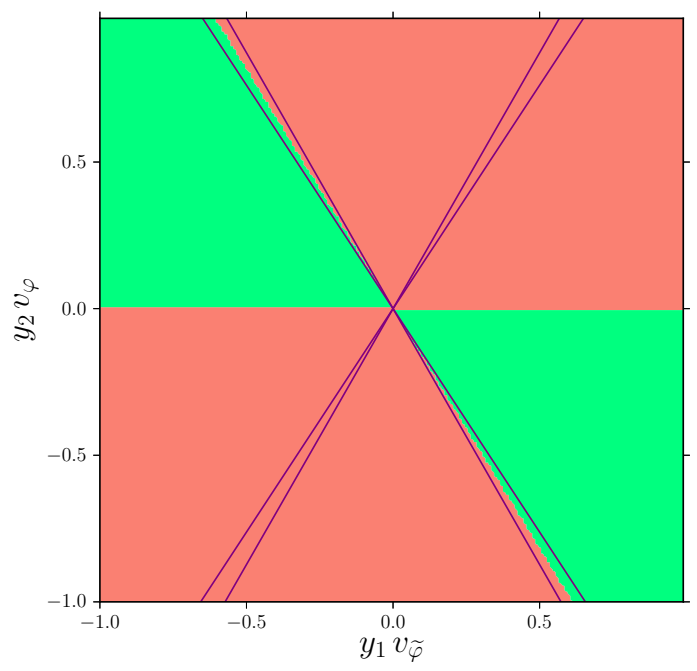

Figure 1. Contour lines of $\Delta m_{31}^{2} / \Delta m_{21}^{2} \equiv 30$ as a function of $y_{1} v_{\widetilde{\varphi}}$ and $y_{2} v_{\varphi}$ for normal neutrino mass ordering. The green region corresponds to values of the parameters where the mixing angles are tribimaximal.

We present the results in figure 2(a). The LO order prediction has given way to a "blob" centered around the tribimaximal values for the mixing angles. Remarkably, 99\% of the points lie in the $3 \sigma$ interval [33] of $\theta_{23}$, whereas $13 \%$ also satisfy the $3 \sigma$ bounds for $\theta_{12}$ (see first panel of figure 2(a)). As was to be expected, the strongest constraint comes from $\theta_{13}$. From the third panel of figure 2(a) we see that low values for $\theta_{13}$ are preferred, but $10 \%$ of the points can accommodate a large reactor angle in agreement with experiment. This number drops to $1 \%$ when we take the constraints from the other two angles into account.

In figure 2(b) we present the results for a model where all terms except $C_{5}^{\nu}$ that lead to departures from tribimaximal mixing are suppressed. This will correspond to the case of the renormalizable model that we will discuss in section 3. As we can see from the first panel of figure $2(\mathrm{~b}), \theta_{12}$ is practically unchanged from its tribimaximal value, whereas $\theta_{23}$ varies very little, and both angles are within their $3 \sigma$ error bands. At the same time, we can easily obtain a large $\theta_{13}$ in such a way that the experimental constraints on the other two angles are always satisfied (blue points in the third panel of figure 2(b)). At the same time we can observe an interesting correlation between $\theta_{23}$ and $\theta_{13}$, namely the larger $\theta_{13}$ is, the more $\theta_{23}$ deviates from maximal mixing. ${ }^{3}$ Unfortunately, this effect is symmetric around $\theta_{23}=45^{\circ}$ and hence does not give a hint whether this deviation is positive or negative.

\section{An ultraviolet completion of the model}

We have shown in section 2 how the minimal $T_{7}$ model, which predicts exact tribimaximal lepton mixing at leading order, receives in general significant corrections at next-to-leading

\footnotetext{
${ }^{3}$ This numerical observation is a reflection of a well-known mixing sum rule relating the deviations of the atmospheric and reactor angles from their tribimaximal values via the CP phase $\delta$ [38, 39].
} 

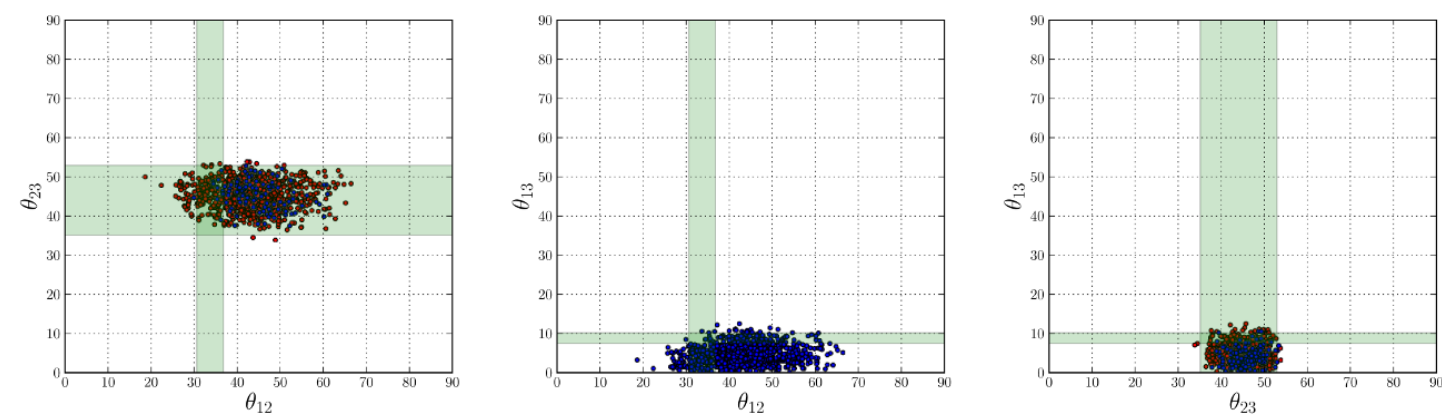

(a) $v_{\varphi} / \Lambda=v_{\widetilde{\varphi}} / \Lambda=0.10$ and $-2.5 \leq \mathfrak{R e} C_{i}^{\alpha}, \mathfrak{I m} C_{i}^{\alpha} \leq 2.5$.
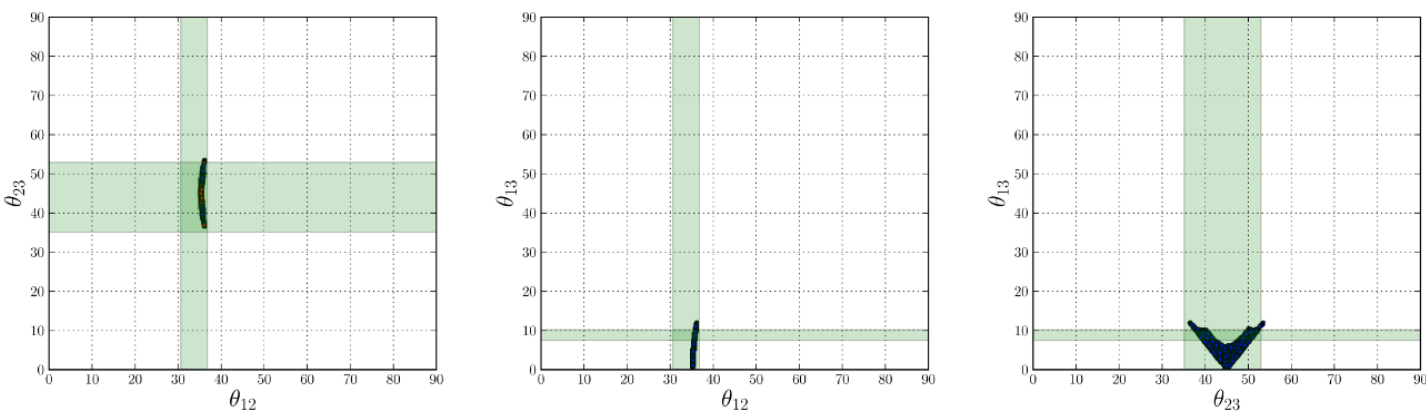

(b) $v_{\varphi} / \Lambda=0.25, v_{\widetilde{\varphi}} / \Lambda=0.05,-2 \leq \mathfrak{R e} C_{5}^{\nu}, \mathfrak{I m} C_{5}^{\nu} \leq 2$, all other coefficients set to zero.

Figure 2. The mixing angles for a given set of leading order coefficients $y_{1}, y_{2}, y_{e}, y_{\mu}, y_{\tau}$ and 1000 sets of random values for the next-to-leading order coefficients $C_{1}^{\nu}, \ldots, C_{3}^{\tau}$. Our choice for the coefficients $y_{1}=0.50, y_{2}=-1.52 y_{1} v_{\tilde{\varphi}} / v_{\varphi}$ corresponds to $\Delta m_{31}^{2} / \Delta m_{21}^{2} \sim 30$ in the LO approximation. The green bands correspond to the $3 \sigma$ intervals [33] for the mixing angles (normal hierarchy in the case of $\theta_{23}$ and $\left.\theta_{13}\right)$. In each diagram, the color of the markers indicates whether the data point lies in the $3 \sigma$ interval of the respective angle that is not plotted (blue) or not (red).

order. Such deviations from the tribimaximal pattern are welcome in order to accommodate the observed large value of the reactor angle $\theta_{13}$. On the other hand, the same set of corrections can potentially modify the other two mixing angles to values disfavored by current global fits. In particular, the solar angle should obtain only small corrections, as its tribimaximal value of $\theta_{12} \approx 35.3^{\circ}$ fits the experimental result already remarkably well. In order to obtain a deviation from tribimaximal mixing which stabilizes the solar angle in first approximation, one can make use of corrections of the so-called trimaximal type [40-48]. These are typically defined by corrections to the tribimaximal neutrino mass matrix whose eigenvectors are proportional to either $(1,1,1)^{T}[39]$ or $(2,-1,-1)^{T}[49]$. Comparison with eq. (2.12) reveals that there exists exactly one NLO correction which respects the trimaximal structure but breaks the tribimaximal one. This subleading contribution to the neutrino mass matrix is proportional to the coefficient $C_{5}^{\nu}$ and can be traced back to an NLO operator in eq. (2.10) in which $L L$ is contracted to a $\mathbf{3}$ of $T_{7}$. Notice that the other two terms with $L L$ contracted to a $\mathbf{3}$, i.e. those proportional to $C_{1}^{\nu}$ and $C_{3}^{\nu}$, do not lead to deviations from the tribimaximal structure. 


\begin{tabular}{|c||c||c|c||c|c|c|c|}
\hline Field & $\Delta$ & $\Theta$ & $\Theta^{c}$ & $\Sigma$ & $\Sigma^{c}$ & $\Omega$ & $\Omega^{c}$ \\
\hline$T_{7}$ & $\mathbf{1}$ & $\mathbf{3}$ & $\overline{\mathbf{3}}$ & $\mathbf{3}$ & $\overline{\mathbf{3}}$ & $\mathbf{3}$ & $\overline{\mathbf{3}}$ \\
\hline $\mathrm{U}(1)_{Y}$ & 2 & -2 & 2 & -2 & 2 & 1 & -1 \\
\hline $\mathrm{U}(1)_{R}$ & 0 & 1 & 1 & 2 & 0 & 1 & 1 \\
\hline
\end{tabular}

Table 2. The charge assignments of the (effective) Higgs $\mathrm{SU}(2)_{L}$ triplet $\Delta$ as well as of the messenger fields required for the ultraviolet completion of the minimal $T_{7}$ model.

This begs the question of how to remove or suppress the unwanted NLO operators while keeping the one proportional to $C_{5}^{\nu}$. To this end, it is useful to recall that we have so far only discussed the model at the effective level, i.e. writing down all (non-renormalizable) terms which are allowed by the imposed symmetries. However, any particular ultraviolet completion of such an effective model does not give rise to all effective NLO terms. The underlying renormalizable model will involve messenger fields which have to be introduced to mediate the effective leading order operators. These necessary messengers typically do not generate all NLO terms [50]. If one particular NLO term is desired, as is the case in our $T_{7}$ model, an extra messenger has to be introduced. In the following we adopt the strategy of considering an ultraviolet completion of the effective $T_{7}$ model such that the operator proportional to $C_{5}^{\nu}$ is allowed, while the bothersome NLO terms are forbidden or sufficiently suppressed. We begin the discussion of the ultraviolet completion of the model with the charged lepton sector, before turning to the more interesting neutrino sector which will be responsible for the breaking of the tribimaximal to the trimaximal pattern.

\subsection{The charged lepton sector}

The leading order operators in eq. (2.1) can be obtained by imposing a $T_{7}$ triplet messenger pair $\Theta, \Theta^{c}$ with the transformation properties as given in table 2. The resulting renormalizable charged lepton superpotential takes the form

$$
W_{\ell}^{\mathrm{ren}} \sim L h_{d} \Theta^{c}+\Theta \widetilde{\varphi} e^{c}+\Theta \widetilde{\varphi} \mu^{c}+\Theta \widetilde{\varphi} \tau^{c}+\Theta \Theta^{c}\left(M_{\Theta}+\varphi+\widetilde{\varphi}\right),
$$

where we have suppressed all dimensionless coupling coefficients. Note that $\Theta$ and $\Theta^{c}$ allow for a bilinear mass term. However, due to the absence of any shaping symmetry, we can also couple a flavon triplet to this product, thus leading to a trilinear term which, after family symmetry breaking, gives a correction to the messenger mass. This is a general feature for heavy messenger fields which transform as triplets under $T_{7}$. Ignoring the correction to the messenger mass, we can integrate out the pair $\Theta, \Theta^{c}$ and obtain the effective superpotential of eq. (2.1) without any higher order corrections. Hence, in the present ultraviolet completion, the only source of deviations from a diagonal charged lepton mass matrix originates from the aforementioned trilinear terms correcting the messenger mass. Such a correction can be rendered sufficiently small by assuming $\langle\widetilde{\varphi}\rangle,\langle\varphi\rangle \ll M_{\Theta}$. In the following, we will therefore ignore any NLO terms in the charged lepton sector. 


\subsection{The neutrino sector}

We now turn to the neutrino sector where we want to formulate a renormalizable theory which, at leading order, gives rise to the effective superpotential in eq. (2.2). The most popular possibility to derive the Weinberg operator from renormalizable terms is provided by the famous type I seesaw mechanism [15-18]. However, applied to the minimal $T_{7}$ model without any shaping symmetry, the type I seesaw mechanism does not yield the tribimaximal structure at leading order. This can be traced back to the fact that the triplet representations of $T_{7}$ are complex. Putting the $L$ into the $\mathbf{3}$ would entail to introduce right-handed neutrinos $N^{c}$ in the $\overline{\mathbf{3}}$ so that the Dirac neutrino Yukawa term $L h_{u} N^{c}$ leads to a Dirac mass matrix that is proportional to the identity matrix. Then the tribimaximal structure of the effective light neutrino mass matrix would be inherited directly from a tribimaximal right-handed neutrino mass matrix $M_{R}$. With $N^{c}$ being a $\overline{\mathbf{3}}$ rather than a $\mathbf{3}$, the alignments of the $\overline{\mathbf{3}}$ flavon $\widetilde{\varphi}$ and the $\mathbf{3}$ flavon $\varphi$ would have to be exchanged to get $M_{R}$ of tribimaximal form. However, changing the alignment of the flavon $\widetilde{\varphi}$ coupling to the charged leptons to $(1,1,1)^{T}$ destroys the diagonal charged lepton mass matrix in eq. (2.3). As a result, under the fairly general set of assumptions that we made above, the total leading order lepton mixing would no longer feature the tribimaximal pattern.

As an alternative to the type I seesaw mechanism, we can adopt the type II seesaw [51-55]. For the sake of clarity, we assume an $\mathrm{SU}(2)_{L}$ triplet Higgs $\Delta$ in the remainder of this section. It is straightforward to replace such a Higgs field by the product of two $h_{u}$ doublets which couple to the square of the lepton doublet $L$ via an $\mathrm{SU}(2)_{L}$ triplet messenger field. The type II seesaw mechanism seems particularly suited for our purposes as we wish to obtain the NLO correction to the neutrino mass matrix proportional to the coupling constant $C_{5}^{\nu}$, which arises from contracting $L L$ to a $\mathbf{3}$ of $T_{7}$. The existence of the effective NLO term $\frac{1}{\Lambda^{3}}(L L)_{\mathbf{3}} h_{u} h_{u} \varphi \widetilde{\varphi}$ in eq. (2.10) requires a messenger field $\Sigma^{c}$ in the $\overline{3}$ representation which couples to the square of the lepton doublet via the renormalizable term $L L \Sigma^{c}$. With the messenger field transforming as a $\overline{\mathbf{3}}$ of $T_{7}$, the product $L L$ is necessarily contracted to a 3 of $T_{7}$. The $\mathrm{U}(1)_{Y}$ and $\mathrm{U}(1)_{R}$ charges required for the existence of $L L \Sigma^{c}$ are given in table 2. As $\Sigma^{c}$ carries e.g. non-zero hypercharge, the messenger cannot be a Majorana particle. In order to allow for a bilinear messenger mass term, we have to invoke a second superfield $\Sigma$, whose charge assignments are fixed by demanding $\Sigma \Sigma^{c}$ to be invariant under all symmetries. The field $\Sigma$ can couple to the Higgs field $\Delta$ and the flavon $\widetilde{\varphi}$, thereby generating the leading order contribution to $M_{\nu}$ proportional to $y_{1}$, cf. eq. (2.4). A similar coupling to the other flavon $\varphi$ is forbidden by the $T_{7}$ family symmetry. We are therefore forced to introduce another pair of messengers $\Omega, \Omega^{c}$, which allows to generate the second leading order contribution to $M_{\nu}$ proportional to $y_{2}$, cf. eq. (2.4). Their charge assignments are again given in table 2 .

With this particle content, the renormalizable neutrino superpotential reads

$$
\begin{aligned}
W_{\nu}^{\mathrm{ren}} \sim & L L \Sigma^{c}+\Sigma \widetilde{\varphi} \Delta+\Sigma \Sigma^{c}\left(M_{\Sigma}+\varphi+\widetilde{\varphi}\right) \\
& +L \varphi \Omega+L \widetilde{\varphi} \Omega+\Omega^{c} \Delta L+\Omega \Omega^{c}\left(M_{\Omega}+\varphi+\widetilde{\varphi}\right) \\
& +L \Sigma^{c} \Omega^{c}+\Sigma^{c} \Omega^{c} \Omega^{c}
\end{aligned}
$$



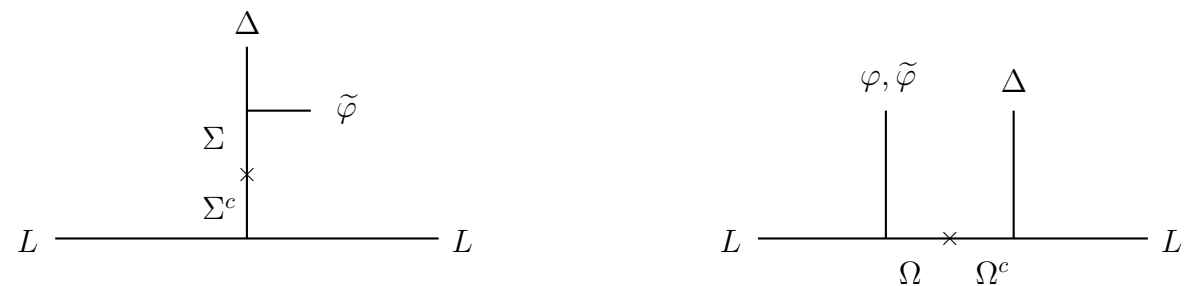

Figure 3. The diagrams contributing to the leading order neutrino superpotential of eq. (2.2).
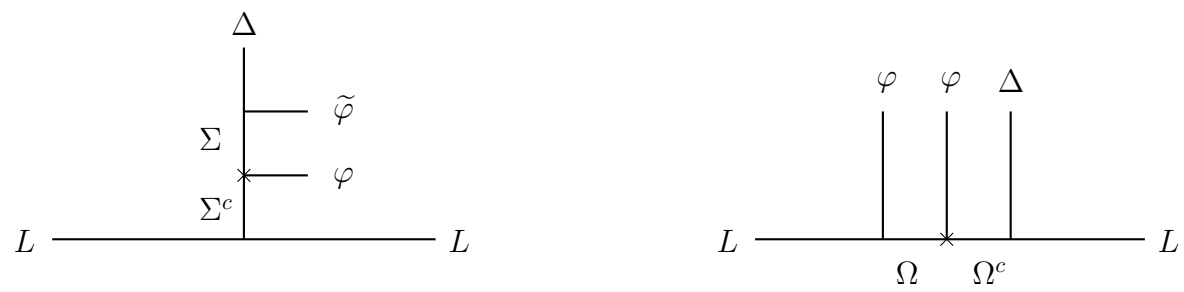

Figure 4. Two diagrams contributing to the NLO neutrino superpotential. The diagram on the left gives the desired trimaximal correction, while the diagram on the right should be suppressed in order to avoid large deviations of the solar angle from its tribimaximal value of $\theta_{12} \approx 35.3^{\circ}$.

where we have suppressed all dimensionless coupling constants. As already discussed for the charged lepton sector, the masses of the $T_{7}$ triplet messengers receive corrections from the flavon vevs. These will break the tribimaximal structure at higher order. Ignoring the corrections to the messenger masses as well as the two operators in the third line of eq. (3.2) results in the effective superpotential of eq. (2.2). The corresponding diagrams are sketched in figure 3 , where the right diagram contributes to the term proportional to $y_{2}$, while the term proportional to $y_{1}$ arises from both diagrams.

We point out that the left diagram in figure 3 is needed only for generating the desired trimaximal NLO correction while suppressing other unwanted NLO terms. In other words, if we wanted to end up with tribimaximal neutrino mixing, the diagram with the $\Omega$ messenger would have been perfectly sufficient. In that case we would have to impose the condition $\langle\widetilde{\varphi}\rangle,\langle\varphi\rangle \ll M_{\Omega}$, similar to the situation in the charged lepton sector. However, since accurate tribimaximal mixing is ruled out by the Daya Bay and Reno measurements, we are forced to consider sizable NLO corrections. Due to the structure of the diagram with the $\Omega$ messenger, the NLO terms obtained from attaching a flavon to the cross representing the mass term $M_{\Omega} \Omega \Omega^{c}$ would give rise to corrections that would shift the solar angle away from its experimentally allowed region. Hence, the trimaximal NLO correction must originate from another diagram, namely the one with the $\Sigma$ messenger, more precisely, the left diagram of figure 3 with the flavon $\varphi$ attached to the cross. The resulting diagram is sketched in figure 4, where one NLO diagram involving the $\Omega$ messenger is also shown. The NLO diagram on the right can be suppressed compared to the NLO diagram on the 
left by assuming a hierarchy in the messenger masses. This can be parameterized by

$$
M_{\Sigma} \sim \epsilon^{k} M_{\Omega}
$$

where $k$ is a positive integer and $\epsilon$ an expansion parameter around 0.2. With this hierarchy of messenger masses imposed we also have to assume a hierarchy in the two flavons vevs,

$$
\frac{v_{\widetilde{\varphi}}}{v_{\varphi}} \sim \frac{M_{\Sigma}}{M_{\Omega}} \sim \epsilon^{k}
$$

so that the leading order mass contributions derived from the two diagrams in figure 3 are of similar size. Note that the diagram on the right involving the flavon $\widetilde{\varphi}$ gives a subdominant contribution which is of the same tribimaximal structure as the one obtained form the left diagram. Finally, we need to guarantee that the trimaximal NLO correction of the left diagram in figure 4 is sizable enough to account for the observed value of reactor angle $\theta_{13}$. This requires the correction to the mass of the $\Sigma$ messenger originating from the flavon $\varphi$ to be of order $\epsilon$, i.e.

$$
\frac{v_{\varphi}}{M_{\Sigma}} \sim \epsilon
$$

Defining $M \sim M_{\Omega}$ we can summarize the requirements on the hierarchies as

$$
v_{\widetilde{\varphi}} \sim \epsilon^{2 k+1} M, \quad v_{\varphi} \sim \epsilon^{k+1} M, \quad M_{\Sigma} \sim \epsilon^{k} M, \quad M_{\Omega} \sim M,
$$

yielding neutrino mass contributions proportional to $\frac{v_{\widetilde{\varphi}}}{M_{\Sigma}} \sim \frac{v_{\varphi}}{M_{\Omega}} \sim \epsilon^{k+1}$ at leading order, with a trimaximal NLO correction proportional to $\epsilon^{k+2}$. In comparison, the right diagram in figure 4 gives a contribution proportional to $\epsilon^{2 k+2}$ which is suppressed by a factor of $\epsilon^{k}$ relative to the desired NLO correction.

With the above assumptions on the hierarchies it is possible to suppress all unwanted contractions in eq. (2.10) while keeping the desired one proportional to $C_{5}^{\nu}$. This holds true even if we take into consideration possible higher order diagrams obtained from the two terms of the third line of eq. (3.2). These terms can give rise to diagrams which involve one $\Sigma$ messenger as well as one or two $\Omega$ messengers. Multiplying the appropriate flavon fields, one can easily find that the maximal contribution derived from the renormalizable term $L \Sigma^{c} \Omega^{c}$ is of order $\epsilon^{2 k+2}$, while the one derived from $\Sigma^{c} \Omega^{c} \Omega^{c}$ is of order $\epsilon^{3 k+3}$. This shows that these higher order corrections are also suppressed with respect to the trimaximal NLO correction by at least a factor of $\epsilon^{k}$.

We conclude this section by mentioning that we have also checked these results numerically for the case of $k=2$. Integrating out the messengers we have first determined the effective neutrino mass matrix, from which we have calculated the mixing angles using the Mixing Parameter Tools provided with the REAP package [56]. This numerical check confirms the results of this section, showing how an ultraviolet completion of the minimal $T_{7}$ model can lead to deviations from tribimaximal mixing which are compatible with a sizable reactor angle as well as a solar angle that is close to its tribimaximal value. 


\section{Vacuum alignment}

\subsection{General discussion}

Any model of flavor that tries to explain a particular mixing pattern by means of a family symmetry which gets broken by flavon vevs has to justify the imposed alignments. In this section we study the possibilities of achieving the vacuum configuration required by the minimal $T_{7}$ model. Following the idea of [23], we demand that supersymmetry be unbroken at the scale of family symmetry breaking. This so-called $F$-term alignment mechanism introduces driving fields whose $F$-terms are set to zero, thus entailing the important $F$-term conditions. Just like the regular flavons, driving fields are neutral under the $\mathrm{SU}(2)_{L} \times \mathrm{U}(1)_{Y}$ gauge symmetry. With the five irreducible representations of $T_{7}$ and no $\mathbb{Z}_{N}$ shaping symmetry, one can introduce driving fields in at most five different representations. Hence, we obtain five distinct $F$-term conditions which we discuss in the following. Throughout this section we will drop all dimensionless coupling as well as overall CG coefficients.

A driving field $D_{1}$ transforming in the 1 of $T_{7}$ allows for two invariant terms in the renormalizable flavon superpotential

$$
W_{\text {flav }} \supset D_{1}\left(M^{2}+\widetilde{\varphi} \varphi\right),
$$

which lead to the $F$-term condition

$$
\frac{\partial W_{\text {flav }}}{\partial D_{1}}=M^{2}+\left\langle\widetilde{\varphi}_{1}\right\rangle\left\langle\varphi_{1}\right\rangle+\left\langle\widetilde{\varphi}_{2}\right\rangle\left\langle\varphi_{2}\right\rangle+\left\langle\widetilde{\varphi}_{3}\right\rangle\left\langle\varphi_{3}\right\rangle=0 .
$$

Similarly, the driving fields $D_{\mathbf{r}}$ in the representations $\mathbf{r}=\mathbf{1}^{\prime}, \mathbf{1}^{\prime \prime}, \mathbf{3}, \overline{\mathbf{3}}$ of $T_{7}$ lead to the conditions:

$$
\begin{aligned}
& \frac{\partial W_{\text {flav }}}{\partial D_{1^{\prime}}}=\left\langle\widetilde{\varphi}_{1}\right\rangle\left\langle\varphi_{2}\right\rangle+\left\langle\widetilde{\varphi}_{2}\right\rangle\left\langle\varphi_{3}\right\rangle+\left\langle\widetilde{\varphi}_{3}\right\rangle\left\langle\varphi_{1}\right\rangle=0, \\
& \frac{\partial W_{\text {flav }}}{\partial D_{1^{\prime \prime}}}=\left\langle\widetilde{\varphi}_{1}\right\rangle\left\langle\varphi_{3}\right\rangle+\left\langle\widetilde{\varphi}_{2}\right\rangle\left\langle\varphi_{1}\right\rangle+\left\langle\widetilde{\varphi}_{3}\right\rangle\left\langle\varphi_{2}\right\rangle=0, \\
& \frac{\partial W_{\text {flav }}}{\partial D_{3}}=M_{1}\left(\begin{array}{c}
\left\langle\widetilde{\varphi}_{1}\right\rangle \\
\left\langle\widetilde{\varphi}_{2}\right\rangle \\
\left\langle\widetilde{\varphi}_{3}\right\rangle
\end{array}\right)+\left(\begin{array}{c}
\left\langle\widetilde{\varphi}_{1}\right\rangle^{2}+2\left\langle\widetilde{\varphi}_{2}\right\rangle\left\langle\widetilde{\varphi}_{3}\right\rangle \\
\omega^{2}\left(\left\langle\widetilde{\varphi}_{3}\right\rangle^{2}+2\left\langle\widetilde{\varphi}_{1}\right\rangle\left\langle\widetilde{\varphi}_{2}\right\rangle\right) \\
\omega\left(\left\langle\widetilde{\varphi}_{2}\right\rangle^{2}+2\left\langle\widetilde{\varphi}_{3}\right\rangle\left\langle\widetilde{\varphi}_{1}\right\rangle\right)
\end{array}\right) \\
& +\left(\begin{array}{l}
\left\langle\varphi_{1}\right\rangle^{2}-\left\langle\varphi_{2}\right\rangle\left\langle\varphi_{3}\right\rangle \\
\left\langle\varphi_{2}\right\rangle^{2}-\left\langle\varphi_{3}\right\rangle\left\langle\varphi_{1}\right\rangle \\
\left\langle\varphi_{3}\right\rangle^{2}-\left\langle\varphi_{1}\right\rangle\left\langle\varphi_{2}\right\rangle
\end{array}\right)+\left(\begin{array}{c}
\left\langle\widetilde{\varphi}_{1}\right\rangle\left\langle\varphi_{1}\right\rangle+\omega\left\langle\widetilde{\varphi}_{2}\right\rangle\left\langle\varphi_{2}\right\rangle+\omega^{2}\left\langle\widetilde{\varphi}_{3}\right\rangle\left\langle\varphi_{3}\right\rangle \\
\left\langle\widetilde{\varphi}_{1}\right\rangle\left\langle\varphi_{3}\right\rangle+\omega\left\langle\widetilde{\varphi}_{2}\right\rangle\left\langle\varphi_{1}\right\rangle+\omega^{2}\left\langle\widetilde{\varphi}_{3}\right\rangle\left\langle\varphi_{2}\right\rangle \\
\left\langle\widetilde{\varphi}_{1}\right\rangle\left\langle\varphi_{2}\right\rangle+\omega\left\langle\widetilde{\varphi}_{2}\right\rangle\left\langle\varphi_{3}\right\rangle+\omega^{2}\left\langle\widetilde{\varphi}_{3}\right\rangle\left\langle\varphi_{1}\right\rangle
\end{array}\right)=\left(\begin{array}{l}
0 \\
0 \\
0
\end{array}\right), \\
& \frac{\partial W_{\mathrm{flav}}}{\partial D_{\overline{\mathbf{3}}}}=M_{2}\left(\begin{array}{c}
\left\langle\varphi_{1}\right\rangle \\
\left\langle\varphi_{2}\right\rangle \\
\left\langle\varphi_{3}\right\rangle
\end{array}\right)+\left(\begin{array}{c}
\left\langle\varphi_{1}\right\rangle^{2}+2\left\langle\varphi_{2}\right\rangle\left\langle\varphi_{3}\right\rangle \\
\omega\left(\left\langle\varphi_{3}\right\rangle^{2}+2\left\langle\varphi_{1}\right\rangle\left\langle\varphi_{2}\right\rangle\right) \\
\omega^{2}\left(\left\langle\varphi_{2}\right\rangle^{2}+2\left\langle\varphi_{3}\right\rangle\left\langle\varphi_{1}\right\rangle\right)
\end{array}\right) \\
& +\left(\begin{array}{l}
\left\langle\widetilde{\varphi}_{1}\right\rangle^{2}-\left\langle\widetilde{\varphi}_{2}\right\rangle\left\langle\widetilde{\varphi}_{3}\right\rangle \\
\left\langle\widetilde{\varphi}_{2}\right\rangle^{2}-\left\langle\widetilde{\varphi}_{3}\right\rangle\left\langle\widetilde{\varphi}_{1}\right\rangle \\
\left\langle\widetilde{\varphi}_{3}\right\rangle^{2}-\left\langle\widetilde{\varphi}_{1}\right\rangle\left\langle\widetilde{\varphi}_{2}\right\rangle
\end{array}\right)+\left(\begin{array}{c}
\left\langle\widetilde{\varphi}_{1}\right\rangle\left\langle\varphi_{1}\right\rangle+\omega^{2}\left\langle\widetilde{\varphi}_{2}\right\rangle\left\langle\varphi_{2}\right\rangle+\omega\left\langle\widetilde{\varphi}_{3}\right\rangle\left\langle\varphi_{3}\right\rangle \\
\left\langle\widetilde{\varphi}_{3}\right\rangle\left\langle\varphi_{1}\right\rangle+\omega^{2}\left\langle\widetilde{\varphi}_{1}\right\rangle\left\langle\varphi_{2}\right\rangle+\omega\left\langle\widetilde{\varphi}_{2}\right\rangle\left\langle\varphi_{3}\right\rangle \\
\left\langle\widetilde{\varphi}_{2}\right\rangle\left\langle\varphi_{1}\right\rangle+\omega^{2}\left\langle\widetilde{\varphi}_{3}\right\rangle\left\langle\varphi_{2}\right\rangle+\omega\left\langle\widetilde{\varphi}_{1}\right\rangle\left\langle\varphi_{3}\right\rangle
\end{array}\right)=\left(\begin{array}{l}
0 \\
0 \\
0
\end{array}\right) .
\end{aligned}
$$

One can easily see that the desired alignments given in eq. (2.5) are inconsistent with eq. (4.3) and eq. (4.4). Eq. (4.5) also does not admit eq. (2.5) as a solution. Turning to 
eq. (4.6), one can substitute $\left\langle\widetilde{\varphi}_{1}\right\rangle=v_{\widetilde{\varphi}},\left\langle\widetilde{\varphi}_{2}\right\rangle=\left\langle\widetilde{\varphi}_{3}\right\rangle=0$ and $\left\langle\varphi_{1}\right\rangle=\left\langle\varphi_{2}\right\rangle=\left\langle\varphi_{3}\right\rangle=\frac{v_{\varphi}}{\sqrt{3}}$ to obtain

$$
\begin{aligned}
\frac{1}{\sqrt{3}} M_{2} v_{\varphi}+v_{\varphi}^{2}+v_{\widetilde{\varphi}}^{2}+\frac{1}{\sqrt{3}} v_{\widetilde{\varphi}} v_{\varphi} & =0, \\
\frac{1}{\sqrt{3}} M_{2} v_{\varphi}+\omega v_{\varphi}^{2}+\frac{1}{\sqrt{3}} \omega^{2} v_{\widetilde{\varphi}} v_{\varphi} & =0, \\
\frac{1}{\sqrt{3}} M_{2} v_{\varphi}+\omega^{2} v_{\varphi}^{2}+\frac{1}{\sqrt{3}} \omega v_{\widetilde{\varphi}} v_{\varphi} & =0 .
\end{aligned}
$$

Since $v_{\varphi}=0$ is not of physical interest, we can cancel off $v_{\varphi}$ as common factor in the second and third equation, which can then be solved easily to obtain $v_{\varphi}=\frac{M_{2}}{\sqrt{3}}$ and $v_{\widetilde{\varphi}}=M_{2}$. Substituting this into the first equation, yields $2 M_{2}^{2}=0$, which in turn would require $M_{2}=0$. Hence, the desired non-trivial alignment is also incompatible with eq. (4.6).

So far we have assumed that all terms in eq. (4.5) and eq. (4.6) are present. However, in extra dimensional models it is possible to envisage a setup in which the two flavons $\widetilde{\varphi}$ and $\varphi$ are localized on different branes $[22,57]$. Then, a given driving field could, in principle, couple exclusively to the $\widetilde{\varphi}$ flavon, while another driving field could couple exclusively to $\varphi$. If both driving fields transform in the $\mathbf{3}$ of $T_{7}$, it is straightforward (though tedious for the alignment of $\widetilde{\varphi}$ ) to verify that the two flavons feature the desired alignments up to $T_{7}$ transformed solutions.

We conclude that the $F$-term conditions of eqs. (4.3)-(4.6) cannot give rise to the alignments of eq. (2.5) without a mechanism that effectively sets some of the coupling constants to zero.

\subsection{Alignment through a hidden flavon sector}

One of the attractive features of the $T_{7}$ model presented in section 2 is that it does not require the introduction of an extra $\mathbb{Z}_{N}$ shaping symmetry to arrive at tribimaximal mixing at leading order. Unfortunately, as we have found in 4.1, the flavons cannot be aligned without setting some coupling constants of the flavon superpotential to zero. If we do not want to resort to extra dimensions, we are forced to extend the minimal $T_{7}$ symmetry by introducing a new symmetry. However, we will show in this section how this can be achieved without assigning non-trivial charges under this new symmetry to the flavons $\tilde{\varphi}$ and $\varphi$, nor to the lepton and Higgs fields.

To this end, it is necessary to introduce new flavons which do not couple to the leptons. In that sense, one might call them "hidden flavons", constituting a sequestered sector of the model. This separation is achieved by assuming these flavons (together with some new driving fields) to be the only fields which transform non-trivially under a hidden $\mathbb{Z}_{N}^{\text {hid }}$ symmetry. The flavons $\widetilde{\varphi}$ and $\varphi$ of the lepton (or visible) sector can then be aligned with respect to those hidden flavons via orthogonality conditions as we discuss now.

In the hidden sector, we introduce four flavons $\chi, \xi^{\prime}, \psi, \widetilde{\zeta}$ which are aligned by virtue of four driving fields $\widetilde{D}_{\chi}, D_{\psi}, O_{\chi \widetilde{\zeta}}, O_{\psi \widetilde{\zeta}}$. Their transformation properties are given in table 3 , where $x, y, z$ are positive integers smaller than $N$. The minimal $\mathbb{Z}_{N}^{\text {hid }}$ symmetry and the corresponding values for $x, y$ and $z$ will be determined at the end of this section. The resulting terms of the renormalizable hidden flavon superpotential read

$$
W_{\text {flav }}^{\text {hid }}=\widetilde{D}_{\chi}\left(\chi \xi^{\prime}+\chi \chi\right)+D_{\psi} \psi \psi+O_{\chi \widetilde{\zeta}} \chi \widetilde{\zeta}+O_{\psi \widetilde{\zeta}} \psi \widetilde{\zeta}
$$




\begin{tabular}{|c||c|c|c|c||c|c|c|c|}
\hline Field & $\chi$ & $\xi^{\prime}$ & $\psi$ & $\widetilde{\zeta}$ & $\widetilde{D}_{\chi}$ & $D_{\psi}$ & $O_{\chi \widetilde{\zeta}}$ & $O_{\psi \widetilde{\zeta}}$ \\
\hline$T_{7}$ & $\mathbf{3}$ & $\mathbf{1}^{\prime}$ & $\mathbf{3}$ & $\overline{\mathbf{3}}$ & $\overline{\mathbf{3}}$ & $\mathbf{3}$ & $\mathbf{1}$ & $\mathbf{1}$ \\
\hline $\mathbb{Z}_{N}^{\text {hid }}$ & $x$ & $x$ & $y$ & $z$ & $-2 x$ & $-2 y$ & $-x-z$ & $-y-z$ \\
\hline $\mathrm{U}(1)_{R}$ & 0 & 0 & 0 & 0 & 2 & 2 & 2 & 2 \\
\hline
\end{tabular}

Table 3. The charge assignments of the hidden sector. $x, y, z$ are positive integers smaller than $N$. The smallest possible value of $N$ is 6 with $(x, y, z)=(2,1,5)$ or equivalently $(4,5,1)$, see discussion at the end of this section.

Setting the $F$-terms of the driving fields to zero gives rise to the conditions

$$
\begin{aligned}
& \frac{\partial W_{\text {flav }}^{\text {hid }}}{\partial \widetilde{D}_{\chi}}=\left(\begin{array}{c}
\left\langle\chi_{2}\right\rangle \\
\left\langle\chi_{3}\right\rangle \\
\left\langle\chi_{1}\right\rangle
\end{array}\right)\left\langle\xi^{\prime}\right\rangle+\left(\begin{array}{c}
\left\langle\chi_{1}\right\rangle^{2}+2\left\langle\chi_{2}\right\rangle\left\langle\chi_{3}\right\rangle \\
\omega\left(\left\langle\chi_{3}\right\rangle^{2}+2\left\langle\chi_{1}\right\rangle\left\langle\chi_{2}\right\rangle\right) \\
\omega^{2}\left(\left\langle\chi_{2}\right\rangle^{2}+2\left\langle\chi_{3}\right\rangle\left\langle\chi_{1}\right\rangle\right)
\end{array}\right)=\left(\begin{array}{l}
0 \\
0 \\
0
\end{array}\right), \\
& \frac{\partial W_{\text {flav }}^{\text {hid }}}{\partial D_{\psi}}=\left(\begin{array}{c}
\left\langle\psi_{1}\right\rangle^{2}-\left\langle\psi_{2}\right\rangle\left\langle\psi_{3}\right\rangle \\
\left\langle\psi_{2}\right\rangle^{2}-\left\langle\psi_{3}\right\rangle\left\langle\psi_{1}\right\rangle \\
\left\langle\psi_{3}\right\rangle^{2}-\left\langle\psi_{1}\right\rangle\left\langle\psi_{2}\right\rangle
\end{array}\right)=\left(\begin{array}{l}
0 \\
0 \\
0
\end{array}\right) \\
& \frac{\partial W_{\text {flav }}^{\text {hid }}}{\partial O_{\chi \widetilde{\zeta}}}=\left\langle\chi_{1}\right\rangle\left\langle\widetilde{\zeta}_{1}\right\rangle+\left\langle\chi_{2}\right\rangle\left\langle\widetilde{\zeta}_{2}\right\rangle+\left\langle\chi_{3}\right\rangle\left\langle\widetilde{\zeta}_{3}\right\rangle=0 \\
& \frac{\partial W_{\text {flav }}^{\text {hid }}}{\partial O_{\psi \widetilde{\zeta}}}=\left\langle\psi_{1}\right\rangle\left\langle\widetilde{\zeta}_{1}\right\rangle+\left\langle\psi_{2}\right\rangle\left\langle\widetilde{\zeta}_{2}\right\rangle+\left\langle\psi_{3}\right\rangle\left\langle\widetilde{\zeta}_{3}\right\rangle=0
\end{aligned}
$$

These conditions can be solved exactly to give a total of 21 sets of solutions for the hidden flavon alignments. A brief description of how these can be derived is outlined below.

First, we consider eq. (4.12). Without loss of generality we can distinguish two cases: $\left\langle\psi_{1}\right\rangle=0$ and $\left\langle\psi_{1}\right\rangle \neq 0$. In the former case one quickly sees that it requires vanishing values for all the components of $\langle\psi\rangle$, thus giving only the trivial vacuum. For $\left\langle\psi_{1}\right\rangle \neq 0$, we obtain three solutions, $\langle\psi\rangle \propto(1,1,1)^{T},\langle\psi\rangle \propto\left(1, \omega^{2}, \omega\right)^{T}$ and $\langle\psi\rangle \propto\left(1, \omega, \omega^{2}\right)^{T}$, which are related to each other by the $T_{7}$ transformation $d$. We remark that a $c$ transformation applied to these three solutions does not modify the alignments but only multiplies them with an irrelevant overall phase.

Next, we turn to eq. (4.11). Again there are two cases to distinguish, $\left\langle\chi_{1}\right\rangle=0$ and $\left\langle\chi_{1}\right\rangle \neq 0$. For the former case the solution is easily found to be $\langle\chi\rangle \propto(0,0,1)^{T}$. With $\left\langle\chi_{1}\right\rangle \neq 0$ on the other hand, it is possible to show that there exist six different solutions, all of which are related to the previous solution by the $T_{7}$ symmetry transformation $c$ : $\langle\chi\rangle \propto c^{k}(0,0,1)^{T}$, where $k=0,1, \ldots, 6$. One can check that applying a $d$ transformation to any of these seven solutions does not yield new alignments.

Combining our results so far, we have 21 different pairs of alignments $\langle\chi\rangle$ and $\langle\psi\rangle$. Being related by the 21 different $T_{7}$ symmetry transformations, they are physically equivalent and one can choose one pair without loss of generality. Having fixed the alignments of $\langle\chi\rangle$ and $\langle\psi\rangle$, eq. (4.13) and eq. (4.14) determine the alignment of $\langle\widetilde{\zeta}\rangle$ uniquely to be 


\begin{tabular}{|c||c|c|c|c|}
\hline Field & $O_{\chi \widetilde{\varphi}}$ & $O_{\chi \widetilde{\varphi}}^{\prime}$ & $O_{\widetilde{\zeta} \varphi}$ & $O_{\widetilde{\zeta} \varphi}^{\prime}$ \\
\hline$T_{7}$ & $\mathbf{1}$ & $\mathbf{1}^{\prime}$ & $\mathbf{1}$ & $\mathbf{1}^{\prime}$ \\
\hline $\mathbb{Z}_{N}^{\text {hid }}$ & $-x$ & $-x$ & $-z$ & $-z$ \\
\hline $\mathrm{U}(1)_{R}$ & 2 & 2 & 2 & 2 \\
\hline
\end{tabular}

Table 4. Charge assignments of the driving fields coupling the hidden flavons $\chi, \widetilde{\zeta}$ to the flavons $\widetilde{\varphi}, \varphi$.

perpendicular to both $\langle\chi\rangle$ and $\langle\psi\rangle$. This shows that in total there exist 21 sets of solutions for the hidden flavon alignments. For convenience we choose the following simple set

$$
\langle\chi\rangle \propto\left(\begin{array}{l}
0 \\
0 \\
1
\end{array}\right), \quad\langle\psi\rangle \propto\left(\begin{array}{l}
1 \\
1 \\
1
\end{array}\right), \quad\langle\widetilde{\zeta}\rangle \propto\left(\begin{array}{c}
1 \\
-1 \\
0
\end{array}\right) .
$$

Let us now turn to the discussion of aligning the original flavon fields $\widetilde{\varphi}$ and $\varphi$ which enter in the lepton sector. Their alignment is dictated by new driving fields which couple the pre-aligned hidden flavons to $\widetilde{\varphi}$ and $\varphi$. The transformation properties of the extra driving fields $O_{\chi \widetilde{\varphi}}, O_{\chi \widetilde{\varphi}}^{\prime}, O_{\widetilde{\zeta} \varphi}$ and $O_{\widetilde{\zeta} \varphi}^{\prime}$ are listed in table 4. With these assignments, the resulting renormalizable flavon superpotential consisting of all $T_{7}$ and $\mathbb{Z}_{N}^{\text {hid }}$ invariant terms reads

$$
W_{\text {flav }}^{\prime}=O_{\chi \widetilde{\varphi} \chi \widetilde{\varphi}}+O_{\chi \widetilde{\varphi}}^{\prime} \chi \widetilde{\varphi}+O_{\widetilde{\zeta} \varphi} \widetilde{\zeta} \varphi+O_{\widetilde{\zeta} \varphi}^{\prime} \widetilde{\zeta} \varphi
$$

The derived $F$-term conditions take the form

$$
\begin{aligned}
& \frac{\partial W_{\text {flav }}^{\prime}}{\partial O_{\chi \widetilde{\varphi}}}=\left\langle\chi_{1}\right\rangle\left\langle\widetilde{\varphi}_{1}\right\rangle+\left\langle\chi_{2}\right\rangle\left\langle\widetilde{\varphi}_{2}\right\rangle+\left\langle\chi_{3}\right\rangle\left\langle\widetilde{\varphi}_{3}\right\rangle=\left\langle\chi_{3}\right\rangle\left\langle\widetilde{\varphi}_{3}\right\rangle=0, \\
& \frac{\partial W_{\text {flav }}^{\prime}}{\partial O_{\chi \widetilde{\varphi}}^{\prime}}=\left\langle\chi_{1}\right\rangle\left\langle\widetilde{\varphi}_{3}\right\rangle+\left\langle\chi_{2}\right\rangle\left\langle\widetilde{\varphi}_{1}\right\rangle+\left\langle\chi_{3}\right\rangle\left\langle\widetilde{\varphi}_{2}\right\rangle=\left\langle\chi_{3}\right\rangle\left\langle\widetilde{\varphi}_{2}\right\rangle=0, \\
& \frac{\partial W_{\text {flav }}^{\prime}}{\partial O_{\widetilde{\zeta} \varphi}}=\left\langle\widetilde{\zeta}_{1}\right\rangle\left\langle\varphi_{1}\right\rangle+\left\langle\widetilde{\zeta}_{2}\right\rangle\left\langle\varphi_{2}\right\rangle+\left\langle\widetilde{\zeta}_{3}\right\rangle\left\langle\varphi_{3}\right\rangle=\left\langle\widetilde{\zeta}_{1}\right\rangle\left(\left\langle\varphi_{1}\right\rangle-\left\langle\varphi_{2}\right\rangle\right)=0, \\
& \frac{\partial W_{\text {flav }}^{\prime}}{\partial O_{\widetilde{\zeta} \varphi}^{\prime}}=\left\langle\widetilde{\zeta}_{3}\right\rangle\left\langle\varphi_{1}\right\rangle+\left\langle\widetilde{\zeta}_{1}\right\rangle\left\langle\varphi_{2}\right\rangle+\left\langle\widetilde{\zeta}_{2}\right\rangle\left\langle\varphi_{3}\right\rangle=\left\langle\widetilde{\zeta}_{1}\right\rangle\left(\left\langle\varphi_{2}\right\rangle-\left\langle\varphi_{3}\right\rangle\right)=0,
\end{aligned}
$$

yielding the desired alignments, cf. eq. (2.5),

$$
\langle\widetilde{\varphi}\rangle \propto\left(\begin{array}{l}
1 \\
0 \\
0
\end{array}\right), \quad\langle\varphi\rangle \propto\left(\begin{array}{l}
1 \\
1 \\
1
\end{array}\right) .
$$

Finally, we need to choose an appropriate $\mathbb{Z}_{N}^{\text {hid }}$, i.e. suitable values for $N, x, y$ and $z$, so that no additional renormalizable terms, other than the ones discussed in this section, occur in the flavon superpotential of eq. (4.10) and eq. (4.16). Through a systematic search, 
we find that the smallest $\mathbb{Z}_{N}^{\text {hid }}$ that we can have is a $\mathbb{Z}_{6}^{\text {hid }}$ [although eq. (4.10) alone can be obtained using a $\left.\mathbb{Z}_{5}^{\text {hid }}\right]$. For a $\mathbb{Z}_{6}^{\text {hid }}$, we can have $(x, y, z)$ as $(2,1,5)$ or $(4,5,1)$. Note that these two choices are equivalent solutions since the elements of the group corresponding to one set are the negatives of the elements corresponding to the other.

\section{Conclusions}

In the present work, we discussed a minimal model of neutrino flavor. In this context, the term "minimal" refers to the order of our flavor group $T_{7}$, and to the number of flavon fields. It is interesting to note that the $\mathbb{Z}_{2} \times \mathbb{Z}_{2}$ Klein symmetry is not a subgroup of $T_{7}$, but arises completely accidentally. As such, it is - to the best of our knowledge - the first indirect model in which the flavon fields appear linearly (and not quadratically) in the leading order structure of the model. Another striking feature of our model is the absence of any Abelian shaping symmetries like $\mathbb{Z}_{N}$ or U(1) in the Yukawa sector. As a consequence, our flavor group $T_{7}$ with its 21 elements is smaller than any symmetry $A_{4} \times \mathbb{Z}_{\mathbb{N}}$ that has long been a paradigm for model building before Daya Bay's and Reno's measurements of a large reactor angle $\theta_{13} \simeq 9^{\circ}$. Our model predicts tribimaximal mixing at leading order, and we achieve a sizable $\theta_{13}$ by considering next-to-leading order corrections to the superpotential. It turns out that models in full agreement with experiment can be obtained for some generic values of the flavon vevs and coefficients in the superpotential. More elegantly, however, one can isolate the next-to-leading order contribution proportional to the coefficient $C_{5}^{\nu}$. Being of trimaximal type, this correction drives $\theta_{13}$ to sizable values while leaving the solar angle very close to its tribimaximal value. The atmospheric mixing angle is correlated to the size of $\theta_{13}$ and stays within the allowed $3 \sigma$ region. In section 3 , we embedded our effective theory into a renormalizable model that naturally suppresses all next-to-leading order contributions except the trimaximal one which is responsible for the large reactor angle. In the last section, we discussed how the flavon fields can be dynamically aligned to yield the required symmetry breaking pattern. The alignment of the flavon fields necessitated the introduction of an Abelian shaping symmetry and so-called driving fields, thereby spoiling the minimality of our model. The smallest symmetry that is viable turns out to be $\mathbb{Z}_{6}$, and as such, our flavor group $T_{7} \times \mathbb{Z}_{6}$ is larger than $A_{4} \times \mathbb{Z}_{N}$ for $N \leq 10$. However, one should note that the $F$-term alignment we considered in section 4 is only one possible option and that the details of an elegant and simple mechanism of vacuum stabilization are still lurking in the dark.

\section{Acknowledgments}

We acknowledge useful discussions with Steve King and Stuart Raby. C.L. acknowledges partial support from the EU ITN grants UNILHC 237920 and INVISIBLES 289442 and would like to thank the LPSC Grenoble for their hospitality. K.P. is supported by the Shyama Prasad Mukherjee Fellowship from the Council of Scientific and Industrial Research (CSIR), India. C.L. and A.W. would like to thank the Galileo Galilei Institute for Theoretical Physics in Florence for their hospitality. We are greatly indebted to the Centre de Calcul de l'Institut National de Physique Nucléaire et Physique des Particules in Lyon for using their resources. 


\begin{tabular}{|cccc|}
\hline & $d$ & $c$ \\
\hline $\mathbf{1}$ & 1 & 1 & \\
$\mathbf{1}^{\prime}$ & $\omega$ & 1 & \\
$\mathbf{1}^{\prime \prime}$ & $\omega^{2}$ & 1 & \\
$\mathbf{3}$ & $\left(\begin{array}{ccc}1 & 0 & 0 \\
0 & \omega^{2} & 0 \\
0 & 0 & \omega\end{array}\right)$ & $\frac{\eta}{3}\left(\begin{array}{ccc}1+\eta+\eta^{3} & \omega^{2}+\omega \eta+\eta^{3} & \omega+\omega^{2} \eta+\eta^{3} \\
\omega+\omega^{2} \eta+\eta^{3} & 1+\eta+\eta^{3} & \omega^{2}+\omega \eta+\eta^{3} \\
\omega^{2}+\omega \eta+\eta^{3} & \omega+\omega^{2} \eta+\eta^{3} & 1+\eta+\eta^{3}\end{array}\right)$ \\
$\overline{\mathbf{3}}\left(\begin{array}{ccc}1 & 0 & 0 \\
0 & \omega & 0 \\
0 & 0 & \omega^{2}\end{array}\right)$ & $\frac{\eta^{6}}{3}\left(\begin{array}{ccc}1+\eta^{6}+\eta^{4} & \omega+\omega^{2} \eta^{6}+\eta^{4} & \omega^{2}+\omega \eta^{6}+\eta^{4} \\
\omega^{2}+\omega \eta^{6}+\eta^{4} & 1+\eta^{6}+\eta^{4} & \omega+\omega^{2} \eta^{6}+\eta^{4} \\
\omega+\omega^{2} \eta^{6}+\eta^{4} & \omega^{2}+\omega \eta^{6}+\eta^{4} & 1+\eta^{6}+\eta^{4}\end{array}\right)$ \\
\hline
\end{tabular}

Table 5. The $T_{7}$ generators of the five irreducible representations in the basis where $d$ is diagonal. Here we have defined $\omega=e^{\frac{2 \pi i}{3}}$ and $\eta=e^{\frac{2 \pi i}{7}}$.

\section{A Generators and Clebsch-Gordan coefficients of $\boldsymbol{T}_{\mathbf{7}}$}

In this appendix we list the relevant group theory of $T_{7}$, which is sometimes also called the Frobenius group $\mathbb{Z}_{7} \rtimes \mathbb{Z}_{3}$. The group is obtained from two generators $c, d$ obeying the presentation, see e.g. [26],

$$
<c, d \mid c^{7}=d^{3}=1, d^{-1} c d=c^{4}>
$$

It has 21 elements and five irreducible representations, namely $\mathbf{1}, \mathbf{1}^{\prime}, \mathbf{1}^{\prime \prime}, \mathbf{3}$, and $\overline{\mathbf{3}}$. A pair of triplet generators satisfying the presentation in A.1 can be found e.g. in [14, 26, 27], where the order-seven generator $c$ is diagonal. For the purpose of the model in the present paper, it is however more convenient to work in a basis with a diagonal order-three generator $d$. Our choice of $T_{7}$ generators for all five irreducible representations is listed in table 5 , where we have defined

$$
\omega=e^{\frac{2 \pi i}{3}}, \quad \eta=e^{\frac{2 \pi i}{7}} .
$$

Although the order-seven generators of the triplet representations look rather involved, the Clebsch-Gordan coefficients take a relatively simple form. Omitting the trivial products, i.e. those involving the singlet $\mathbf{1}$ as well as products of only one-dimensional irreducible representations, the product rules are reported below. We use the convention that the components of the first representation of any given product $\mathbf{a} \otimes \mathbf{b}$ are denoted by $a_{i}$ while we use $b_{i}$ for the components of the second representation. The subscripts $s$ and $a$ stand for "symmetric" and "anti-symmetric", respectively. Note that we have also included the 
normalization factors of the Clebsch-Gordan coefficients.

$$
\begin{aligned}
& \mathbf{1}^{\prime} \otimes \mathbf{3}: \quad \mathbf{3}=a_{1}\left(\begin{array}{l}
b_{2} \\
b_{3} \\
b_{1}
\end{array}\right), \quad \mathbf{1}^{\prime \prime} \otimes \mathbf{3}: \quad \mathbf{3}=a_{1}\left(\begin{array}{l}
b_{3} \\
b_{1} \\
b_{2}
\end{array}\right), \\
& \mathbf{1}^{\prime} \otimes \overline{\mathbf{3}}: \quad \overline{\mathbf{3}}=a_{1}\left(\begin{array}{l}
b_{3} \\
b_{1} \\
b_{2}
\end{array}\right), \quad \mathbf{1}^{\prime \prime} \otimes \overline{\mathbf{3}}: \quad \overline{\mathbf{3}}=a_{1}\left(\begin{array}{l}
b_{2} \\
b_{3} \\
b_{1}
\end{array}\right), \\
& \mathbf{3} \otimes \overline{\mathbf{3}}: \quad \mathbf{1}=\frac{1}{\sqrt{3}}\left(a_{1} b_{1}+a_{2} b_{2}+a_{3} b_{3}\right), \\
& \mathbf{1}^{\prime}=\frac{1}{\sqrt{3}}\left(a_{1} b_{2}+a_{2} b_{3}+a_{3} b_{1}\right), \\
& \mathbf{1}^{\prime \prime}=\frac{1}{\sqrt{3}}\left(a_{1} b_{3}+a_{2} b_{1}+a_{3} b_{2}\right), \\
& \mathbf{3}=\frac{1}{\sqrt{3}}\left(\begin{array}{l}
a_{1} b_{1}+\omega^{2} a_{2} b_{2}+\omega a_{3} b_{3} \\
a_{1} b_{3}+\omega^{2} a_{2} b_{1}+\omega a_{3} b_{2} \\
a_{1} b_{2}+\omega^{2} a_{2} b_{3}+\omega a_{3} b_{1}
\end{array}\right), \\
& \overline{\mathbf{3}}=\frac{1}{\sqrt{3}}\left(\begin{array}{l}
a_{1} b_{1}+\omega a_{2} b_{2}+\omega^{2} a_{3} b_{3} \\
a_{3} b_{1}+\omega a_{1} b_{2}+\omega^{2} a_{2} b_{3} \\
a_{2} b_{1}+\omega a_{3} b_{2}+\omega^{2} a_{1} b_{3}
\end{array}\right), \\
& \mathbf{3} \otimes \mathbf{3}: \quad \mathbf{3}_{s}=\frac{1}{\sqrt{3}}\left(\begin{array}{c}
a_{1} b_{1}+a_{2} b_{3}+a_{3} b_{2} \\
\omega\left(a_{1} b_{2}+a_{2} b_{1}+a_{3} b_{3}\right) \\
\omega^{2}\left(a_{1} b_{3}+a_{2} b_{2}+a_{3} b_{1}\right)
\end{array}\right), \\
& \overline{\mathbf{3}}_{s}=\frac{1}{\sqrt{6}}\left(\begin{array}{c}
2 a_{1} b_{1}-a_{2} b_{3}-a_{3} b_{2} \\
2 a_{2} b_{2}-a_{3} b_{1}-a_{1} b_{3} \\
2 a_{3} b_{3}-a_{1} b_{2}-a_{2} b_{1}
\end{array}\right), \\
& \overline{\mathbf{3}}_{a}=\frac{1}{\sqrt{2}}\left(\begin{array}{l}
a_{2} b_{3}-a_{3} b_{2} \\
a_{3} b_{1}-a_{1} b_{3} \\
a_{1} b_{2}-a_{2} b_{1}
\end{array}\right) \text {. }
\end{aligned}
$$

Open Access. This article is distributed under the terms of the Creative Commons Attribution License which permits any use, distribution and reproduction in any medium, provided the original author(s) and source are credited.

\section{References}

[1] P. Harrison, D. Perkins and W. Scott, Tri-bimaximal mixing and the neutrino oscillation data, Phys. Lett. B 530 (2002) 167 [hep-ph/0202074] [INSPIRE].

[2] P. Harrison and W. Scott, Symmetries and generalizations of tri-bimaximal neutrino mixing, Phys. Lett. B 535 (2002) 163 [hep-ph/0203209] [INSPIRE]. 
[3] Z.-z. Xing, Nearly tri bimaximal neutrino mixing and CP-violation, Phys. Lett. B 533 (2002) 85 [hep-ph/0204049] [INSPIRE].

[4] G. Altarelli and F. Feruglio, Discrete flavor symmetries and models of neutrino mixing, Rev. Mod. Phys. 82 (2010) 2701 [arXiv: 1002.0211] [INSPIRE].

[5] H. Ishimori et al., Non-Abelian discrete symmetries in particle physics, Prog. Theor. Phys. Suppl. 183 (2010) 1 [arXiv:1003.3552] [INSPIRE].

[6] W. Grimus and P.O. Ludl, Finite flavour groups of fermions, J. Phys. A 45 (2012) 233001 [arXiv:1110.6376] [INSPIRE].

[7] C. Hagedorn, S.F. King and C. Luhn, A SUSY GUT of flavour with $S_{4} \times \mathrm{SU}(5)$ to $N L O$, JHEP 06 (2010) 048 [arXiv: 1003.4249] [INSPIRE].

[8] I.K. Cooper, S.F. King and C. Luhn, SUSY SU(5) with singlet plus adjoint matter and $A_{4}$ family symmetry, Phys. Lett. B 690 (2010) 396 [arXiv: 1004.3243] [INSPIRE].

[9] I.K. Cooper, S.F. King and C. Luhn, $A_{4} \times \mathrm{SU}(5)$ SUSY GUT of flavour with trimaximal neutrino mixing, JHEP 06 (2012) 130 [arXiv:1203.1324] [INSPIRE].

[10] C. Hagedorn, S.F. King and C. Luhn, SUSY $S_{4} \times \mathrm{SU}(5)$ revisited, Phys. Lett. B $717(2012)$ 207 [arXiv: 1205.3114] [INSPIRE].

[11] S.F. King, C. Luhn and A.J. Stuart, A Grand $\Delta(96) \times \mathrm{SU}(5)$ flavour model, Nucl. Phys. B 867 (2013) 203 [arXiv: 1207.5741] [INSPIRE].

[12] S. King, Predicting neutrino parameters from $\mathrm{SO}(3)$ family symmetry and quark-lepton unification, JHEP 08 (2005) 105 [hep-ph/0506297] [INSPIRE].

[13] S. King, Parametrizing the lepton mixing matrix in terms of deviations from tri-bimaximal mixing, Phys. Lett. B 659 (2008) 244 [arXiv:0710.0530] [INSPIRE].

[14] S.F. King and C. Luhn, On the origin of neutrino flavour symmetry, JHEP 10 (2009) 093 [arXiv: 0908.1897] [INSPIRE].

[15] P. Minkowski, $\mu \rightarrow$ e $\gamma$ at a Rate of One Out of 1-Billion Muon Decays?, Phys. Lett. B 67 (1977) 421 [INSPIRE].

[16] P. Ramond, The family group in Grand unified theories, hep-ph/9809459 [INSPIRE].

[17] T. Yanagida, Horizontal symmetry and masses of neutrinos, in Proceedings of the Workshop on Unified Theory and Baryon Number of the Universe, KEK, Tsukuba Japan (1979).

[18] R.N. Mohapatra and G. Senjanović, Neutrino mass and spontaneous parity violation, Phys. Rev. Lett. 44 (1980) 912 [INSPIRE].

[19] S. King, Atmospheric and solar neutrinos with a heavy singlet, Phys. Lett. B 439 (1998) 350 [hep-ph/9806440] [INSPIRE].

[20] S. King, Atmospheric and solar neutrinos from single right-handed neutrino dominance and U(1) family symmetry, Nucl. Phys. B 562 (1999) 57 [hep-ph/9904210] [INSPIRE].

[21] S. King, Constructing the large mixing angle MNS matrix in seesaw models with right-handed neutrino dominance, JHEP 09 (2002) 011 [hep-ph/0204360] [INSPIRE].

[22] G. Altarelli and F. Feruglio, Tri-bimaximal neutrino mixing from discrete symmetry in extra dimensions, Nucl. Phys. B 720 (2005) 64 [hep-ph/0504165] [INSPIRE].

[23] G. Altarelli and F. Feruglio, Tri-bimaximal neutrino mixing, $A_{4}$ and the modular symmetry, Nucl. Phys. B 741 (2006) 215 [hep-ph/0512103] [INSPIRE]. 
[24] K.M. Parattu and A. Wingerter, Tribimaximal mixing from small groups, Phys. Rev. D 84 (2011) 013011 [arXiv: 1012.2842] [INSPIRE].

[25] C. Luhn, S. Nasri and P. Ramond, Tri-bimaximal neutrino mixing and the family symmetry semidirect product of $Z_{7} \rtimes Z_{3}$, Phys. Lett. B 652 (2007) 27 [arXiv:0706.2341] [INSPIRE].

[26] C. Luhn, S. Nasri and P. Ramond, Simple finite non-Abelian flavor groups, J. Math. Phys. 48 (2007) 123519 [arXiv:0709.1447] [InSPIRE].

[27] C. Hagedorn, M.A. Schmidt and A.Y. Smirnov, Lepton mixing and cancellation of the Dirac mass hierarchy in $\mathrm{SO}(10)$ GUTs with flavor symmetries $T_{7}$ and $\Sigma(81)$, Phys. Rev. D 79 (2009) 036002 [arXiv:0811.2955] [INSPIRE].

[28] DAYA-BAY collaboration, F. An et al., Observation of electron-antineutrino disappearance at Daya Bay, Phys. Rev. Lett. 108 (2012) 171803 [arXiv:1203.1669] [INSPIRE].

[29] RENO collaboration, J. Ahn et al., Observation of reactor electron antineutrino disappearance in the RENO experiment, Phys. Rev. Lett. 108 (2012) 191802 [arXiv: 1204.0626] [INSPIRE].

[30] S. Weinberg, Baryon and lepton nonconserving processes, Phys. Rev. Lett. 43 (1979) 1566 [INSPIRE].

[31] C. Froggatt and H.B. Nielsen, Hierarchy of quark masses, Cabibbo angles and CP-violation, Nucl. Phys. B 147 (1979) 277 [inSPIRE].

[32] G. Altarelli and D. Meloni, A simplest $A_{4}$ model for tri-bimaximal neutrino mixing, J. Phys. G 36 (2009) 085005 [arXiv:0905.0620] [INSPIRE].

[33] G. Fogli et al., Global analysis of neutrino masses, mixings and phases: entering the era of leptonic CP-violation searches, Phys. Rev. D 86 (2012) 013012 [arXiv: 1205.5254] [INSPIRE].

[34] D. Forero, M. Tortola and J. Valle, Global status of neutrino oscillation parameters after Neutrino-2012, Phys. Rev. D 86 (2012) 073012 [arXiv:1205.4018] [InSPIRE].

[35] M. Gonzalez-Garcia, M. Maltoni, J. Salvado and T. Schwetz, Global fit to three neutrino mixing: critical look at present precision, arXiv:1209.3023 [INSPIRE].

[36] C.I. Low and R.R. Volkas, Tri-bimaximal mixing, discrete family symmetries and a conjecture connecting the quark and lepton mixing matrices, Phys. Rev. D 68 (2003) 033007 [hep-ph/0305243] [INSPIRE].

[37] Particle Data Group collaboration, J. Beringer et al., Review of Particle Physics (RPP), Phys. Rev. D 86 (2012) 010001 [INSPIRE].

[38] S. King, Tri-bimaximal neutrino mixing and $\theta_{13}$, Phys. Lett. B 675 (2009) 347 [arXiv: 0903.3199] [INSPIRE].

[39] S.F. King and C. Luhn, Trimaximal neutrino mixing from vacuum alignment in $A_{4}$ and $S_{4}$ models, JHEP 09 (2011) 042 [arXiv: 1107.5332] [INSPIRE].

[40] N. Haba, A. Watanabe and K. Yoshioka, Twisted flavors and tri/bi-maximal neutrino mixing, Phys. Rev. Lett. 97 (2006) 041601 [hep-ph/0603116] [INSPIRE].

[41] X.-G. He and A. Zee, Minimal modification to the tri-bimaximal neutrino mixing, Phys. Lett. B 645 (2007) 427 [hep-ph/0607163] [INSPIRE].

[42] C. Lam, Mass independent textures and symmetry, Phys. Rev. D 74 (2006) 113004 [hep-ph/0611017] [INSPIRE]. 
[43] W. Grimus and L. Lavoura, A model for trimaximal lepton mixing, JHEP 09 (2008) 106 [arXiv:0809.0226] [INSPIRE].

[44] C.H. Albright and W. Rodejohann, Comparing trimaximal mixing and its variants with deviations from tri-bimaximal mixing, Eur. Phys. J. C 62 (2009) 599 [arXiv:0812.0436] [INSPIRE].

[45] C.H. Albright, A. Dueck and W. Rodejohann, Possible alternatives to tri-bimaximal mixing, Eur. Phys. J. C 70 (2010) 1099 [arXiv: 1004.2798] [InSPIRE].

[46] H. Ishimori, Y. Shimizu, M. Tanimoto and A. Watanabe, Neutrino masses and mixing from $S_{4}$ flavor twisting, Phys. Rev. D 83 (2011) 033004 [arXiv: 1010.3805] [InSPIRE].

[47] Y. Shimizu, M. Tanimoto and A. Watanabe, Breaking tri-bimaximal mixing and large $\theta_{13}$, Prog. Theor. Phys. 126 (2011) 81 [arXiv:1105.2929] [INSPIRE].

[48] X.-G. He and A. Zee, Minimal modification to tri-bimaximal mixing, Phys. Rev. D 84 (2011) 053004 [arXiv: 1106.4359] [INSPIRE].

[49] S. Antusch, S.F. King, C. Luhn and M. Spinrath, Trimaximal mixing with predicted $\theta_{13}$ from a new type of constrained sequential dominance, Nucl. Phys. B 856 (2012) 328 [arXiv: 1108.4278] [INSPIRE].

[50] I. de Medeiros Varzielas and L. Merlo, Ultraviolet completion of flavour models, JHEP 02 (2011) 062 [arXiv:1011.6662] [INSPIRE].

[51] M. Magg and C. Wetterich, Neutrino mass problem and gauge hierarchy, Phys. Lett. B 94 (1980) 61 [INSPIRE].

[52] J. Schechter and J. Valle, Neutrino masses in $\mathrm{SU}(2) \times \mathrm{U}(1)$ theories, Phys. Rev. D 22 (1980) 2227 [INSPIRE].

[53] C. Wetterich, Neutrino masses and the scale of B - L violation, Nucl. Phys. B 187 (1981) 343 [INSPIRE].

[54] G. Lazarides, Q. Shafi and C. Wetterich, Proton lifetime and fermion masses in an $\mathrm{SO}(10)$ model, Nucl. Phys. B 181 (1981) 287 [INSPIRE].

[55] R.N. Mohapatra and G. Senjanović, Neutrino masses and mixings in gauge models with spontaneous parity violation, Phys. Rev. D 23 (1981) 165 [INSPIRE].

[56] S. Antusch, J. Kersten, M. Lindner, M. Ratz and M.A. Schmidt, Running neutrino mass parameters in see-saw scenarios, JHEP 03 (2005) 024 [hep-ph/0501272] [INSPIRE].

[57] B.D. Callen and R.R. Volkas, Large lepton mixing angles from a 4+1-dimensional $\mathrm{SU}(5) \times A_{4}$ domain-wall braneworld model, Phys. Rev. D 86 (2012) 056007 [arXiv: 1205.3617] [INSPIRE]. 